\title{
High-quality seamless DEM generation blending SRTM-1, ASTER GDEM v2 and ICESat/GLAS observations
}

\author{
Linwei Yue ${ }^{[1]}$ Huanfeng Shen ${ }^{[2][3]}$ Liangpei Zhang ${ }^{[1][3]}$ \\ Xianwei Zheng ${ }^{[1]}$ Fan Zhang ${ }^{[1]}$ Qiangqiang Yuan ${ }^{[3][4]}$ \\ ${ }^{[1]}$ The State Key Laboratory of Information Engineering in Surveying, Mapping and Remote \\ Sensing, Wuhan University, Wuhan, P. R. China \\ ${ }^{[2]}$ School of Resource and Environmental Science, Wuhan University, Wuhan, P. R. China \\ ${ }^{[3]}$ Collaborative Innovation Center of Geospatial Technology, Wuhan University, Wuhan, \\ P. R. China \\ ${ }^{[4]}$ School of Geodesy and Geomatics, Wuhan University, Wuhan, P. R. China \\ * Corresponding authors, email: shenhf@whu.edu.cn, zlp62@whu.edu.cn
}

\begin{abstract}
The absence of a high-quality seamless global digital elevation model (DEM) dataset has been a challenge for the Earth-related research fields. Recently, the 1-arc-second Shuttle Radar Topography Mission (SRTM-1) data have been released globally, covering over $80 \%$ of the Earth's land surface $\left(60^{\circ} \mathrm{N}-56^{\circ} S\right)$. However, voids and anomalies still exist in some tiles, which has prevented the SRTM-1 dataset from being directly used without further processing. In this paper, we propose a method to generate a seamless DEM dataset blending SRTM-1, ASTER GDEM v2, and ICESat laser altimetry data. The ASTER GDEM v2 data are used as the elevation source for the SRTM void filling. To get a reliable filling source, ICESat GLAS points are incorporated to enhance the accuracy of the ASTER data within the void regions, using an artificial neural network (ANN) model. After correction, the voids in the SRTM-1 data are filled with the corrected ASTER GDEM values. The triangular irregular network based delta surface fill (DSF) method is then
\end{abstract}


employed to eliminate the vertical bias between them. Finally, an adaptive outlier filter is applied to all the data tiles. The final result is a seamless global DEM dataset. ICESat points collected from 2003 to 2009 were used to validate the effectiveness of the proposed method, and to assess the vertical accuracy of the global DEM products in China. Furthermore, channel networks in the Yangtze River Basin were also extracted for the data assessment.

Keywords: Digital elevation model (DEM); multi-source data fusion; SRTM-1; ASTER GDEM; ICESat/GLAS.

\section{Introduction}

Digital elevation model (DEM) data have been widely applied in scientific fields such as ecology (Kellndorfer et al. 2004; Næsset et al. 2016), agriculture (Fu and Rich 2002), and hydrological modeling (Wechsler 2007; Zheng et al. 2015). With the development of remote sensing and photogrammetric techniques, DEMs now mainly refer to elevation data stored as regularly gridded elevation values, based on remote sensing observations (Robinson et al. 2014).

There are a number of DEM products available for global Earth observation and analysis. The Advanced Spaceborne Thermal Emission and Reflectance Radiometer Global Digital Elevation Model (ASTER GDEM) is a product generated from optical data collected by the ASTER instrument onboard NASA's Terra satellite (Hengl and Reuter 2011). This dataset is the only DEM that covers $\sim 99 \%$ of the entire land surface at a high resolution, but the 
accuracy of the ASTER GDEM has attracted controversy due to the anomalies and noises caused by the limitations of the optical imaging (Tachikawa et al. 2011; Mukherjee et al. 2013). Comparatively, the Shuttle Radar Topography Mission (SRTM) DEM is the most commonly used data source due to its relatively stable accuracy (Yang et al. 2011). This near-global dataset was generated based on spaceborne radar measurements collected in 2000 (Jarvis et al. 2008). However, data voids are common in the mountainous regions with large slope angles due to the squint mode of the SAR imaging (Crosetto 2002; Toutin 2002; Baselice et al. 2009). After void filling, SRTM-3 v4.1 was publicly released with a 1-arc-second $(\sim 30 \mathrm{~m})$ resolution in the U.S and a 3-arc-second $(\sim 90 \mathrm{~m})$ resolution in the rest of the world. More recently, the 1-arc-second global SRTM-1 data product has been released; however, voids still exist in some tiles of complex terrain. Other commonly used global DEM products include the Global 30-Arc-Second Elevation Data Set (GTOPO30) (USGS 1996), the Global Multi-resolution Terrain Elevation Data 2010 (GMTED2010) (USGS 2010), NEXTMAP WORLD $30^{\mathrm{TM}}$ (Tighe and Chamberlain 2009) and WorldDEM $^{\mathrm{TM}}$ (2014). Among them, the 30-m NEXTMAP WORLD $30^{\mathrm{TM}}$ and the 12-m WorldDEM $^{\mathrm{TM}}$ have not yet been freely available to the public. Furthermore, the Geoscience Laser Altimeter System (GLAS) onboard NASA's Ice, Cloud and Land Elevation Satellite (ICESat) provides global laser points with high-accuracy elevation information (Schutz et al. 2005).

There has been extensive research into the assessment of the main public global DEM products (Athmania and Achour 2014; Wang and Wang 2015). Due to the limited spatial resolution of GTOPO30 $(\sim 1 \mathrm{~km})$ and GMTED2010 (30, 15, and 7.5 arc seconds), the two 
datasets cannot satisfy some application demands. Therefore, researchers have mainly focused on the accuracy comparison of the SRTM-3 data and the ASTER GDEM (Hirt et al. 2010; Li et al. 2013; Mukherjee et al. 2013; Satgé et al. 2015; Wang and Wang 2015). The vertical accuracies of both these DEM datasets are closely related to slope and terrain roughness (Toutin 2002). It is generally acknowledged that the SRTM-3 data are superior to ASTER GDEM in terms of vertical accuracy in the low-relief areas (Jacobsen 2010; Zhao et al. 2011; Li et al. 2013), while the ASTER GDEM shows a better performance in some mountainous areas than the SRTM-3 data, which is probably due to the inaccurate void filling of the SRTM-3 data (Li et al. 2013; Yue et al. 2015). The vertical accuracy of ICESat GLAS data points can reach a sub-meter level; however, the sparsely distributed GLAS points are separated by intervals of nearly $170 \mathrm{~m}$ along-track and several kilometers across-track (Zhang et al. 2011).

With the growing demand for the monitoring of Earth surface changes, the absence of a high-quality seamless global DEM dataset has been a challenge for the Earth-related research fields (Robinson et al. 2014). On one hand, modern imaging technologies have been applied to Earth observation, and new DEM products are being generated and released (Fritz et al. 2012; Tadono et al. 2015). On the other hand, the analysis and improvement of the currently available datasets also make sense (Reuter et al. 2007; Arefi and Reinartz 2011; Yue et al. 2012; Robinson et al. 2014). Reuter et al. (2007) presented a void-filling strategy in conjunction with other sources of elevation data using a range of interpolation algorithms. The SRTM-3 v4.1 data were processed following the method described by Reuter et al. (2007). However, the accuracy of the void-filled results are still unstable as 
validated in the related works (Li et al. 2013; Yue et al. 2015). Arefi and Reinartz (2011) tried to utilize ICESat data to improve the accuracy of ASTER GDEM data. Given the sparse distribution of the ICESat data, it is difficult to control the accuracy of the corrected ASTER tiles without sufficient reference points using an ordinary Moving Average interpolation method. Robinson et al. (2014) reconstructed a new 90-m DEM product by integrating multi-scale DEM products (1-arc ASTER GDEM v2, 3-arc SRTM-3 v4.1 and 3-arc GLSDEM) . Nevertheless, they did not fully consider the unstable filling results in SRTM-3 v4.1, and the spatial resolution of the final product is limited (only $90 \mathrm{~m}$ ). In addition, the method only used information from GLSDEM to fill the voids of ASTER GDEM at high latitudes, ignoring the low accuracy of the auxiliary data within the voids.

On the whole, there are two main limitations among these works presented. Firstly, most of the researchers focused on SRTM-3 and ASTER GDEM v2, which are the most popular global DEM datasets. However, the ASTER GDEM is severely affected with random noise and anomalies, while the previously released SRTM-3 v4.1 dataset is limited by the 3 arc second resolution and the inaccurate void-filling results. Secondly, there are few works incorporating the high-quality elevation data in addition to the raster DEM datasets.

Given these facts, the fusion of multi-source and multi-scale data to generate a high-quality seamless DEM product is still challenging. For the first issue, the release of the global 1-arc SRTM-1 dataset can be considered as progress. As voids and anomalies still exist in some tiles, the current SRTM-1 data cannot be directly used without further processing. Nevertheless, with the release of the high-resolution SRTM-1 data, there is now a chance to generate a DEM product with a high resolution, a global extent, and a reliable 
accuracy, by integrating multi-source elevation data. Given the comparative resolutions and the same project coordinate system, the ASTER GDEM v2 can be regarded as a good elevation source for SRTM-1 void filling. Moreover, ICESat laser points can be incorporated to correct the inaccurate elevation values in the ASTER data within the void regions considering the relatively low accuracy in the rugged terrain.

This paper intends to convey a processing method to generate a high-resolution, high-quality, and seamless global DEM product blending the recently released global SRTM-1 dataset, the optical-derived ASTER GDEM v2, and ICESat GLAS data points. To correct the ASTER elevations using the ICESat points, it turns out to be a point-surface fusion problem on account of the large spacing between the ICESat data. The common solution is to generate a correction layer using an interpolation method (Arefi and Reinartz 2011; Verdin et al. 2015), while the ICESat points are used as ground control points (GCPs). Nevertheless, the significant distance between the scanning tracks of the ICESat data results in significant errors. In this paper, we employ an ANN model to simulate the relationship between the ICESat and ASTER GDEM data, followed by a void filling process. Furthermore, an adaptive outlier filter is applied to reduce the anomalies for the non-void areas in the SRTM-1 data.

The rest of the paper is organized as follows. Section 2 gives a detailed description of the data used. In Section 3, we provide the details of the processing flow. The results after void filling and correction are analyzed in Section 4. Finally, Section 5 provides the conclusion.

\section{Datasets}

In this paper, we integrate multi-source elevation data for the generation of a seamless 
global DEM dataset. The data sources include the 1-arc SRTM-1 DEM, the 1-arc ASTER GDEM v2, and the ICESat GLAS land elevation product. Meanwhile, the SRTM-3 DEM data are also included for the accuracy validation and comparison. The specific characteristics of the datasets are provided in the following.

\subsection{SRTM data products}

The SRTM was an international project conducted by NASA and the National Geospatial-Intelligence Agency (NGA) in February 2000 (Van Zyl 2001). The 11-day mission acquired data via radar interferometry using an onboard/outboard antenna system and single-pass data acquisition, which were used to generate near-global land elevation data products. The SRTM was successful in collecting elevation data over $80 \%$ of the Earth's land surface $\left(60^{\circ} N-56^{\circ} S\right)$ (Farr and Kobrick 2000). There are two main global SRTM DEM datasets with different levels of processing.

The original SRTM elevation data were processed from C-band radar signals spaced at intervals of 1 arc seconds at NASA's Jet Propulsion Laboratory (JPL). However, the original data for regions outside the U.S were released at 3 arc seconds for open distribution, and are referred to as the SRTM-3 data. Since its original release, the SRTM-3 dataset has been updated several times for quality improvement. The most commonly used SRTM-3 v4.1 was released by the Consortium for Spatial Information of the Consultative Group of International Agricultural Research (CGIAR-CSI) after data improvement and void filling, and distributed as $5^{\circ} \times 5^{\circ}$ tiles (Jarvis et al. 2008). The vertical error of the DEM was reported to be less than $16 \mathrm{~m}$ at the $90 \%$ confidence level based on the WGS 84 horizontal datum and EGM96 vertical datum (Gamache 2004). 
The SRTM-1 global dataset has been released since September 2014. This newly released dataset provides worldwide coverage of void-filled high-resolution elevation data. However, it still contains large amounts of voids in mountainous regions and areas where the initial processing did not satisfy quality specifications (Pipaud et al. 2015). The SRTM-1 global dataset are distributed as $1^{\circ} \times 1^{\circ}$ tiles.

\subsection{ASTER GDEM v2}

As mentioned before, the ASTER GDEM product was jointly produced by NASA and the Japanese Ministry of Economy, Trade and Industry (METI), based on the measurements collected by NASA's Terra satellite. The original ASTER GDEM1 data were generated employing over 1.2 million observed scenes covering land surfaces from $83^{\circ} \mathrm{N}$ to $83^{\circ} \mathrm{S}$. The overall accuracy of the GDEM1 data is around $20 \mathrm{~m}$ at the $95 \%$ confidence level, as assessed by a joint U.S./Japan validation team (ASTER Validation Team 2009). Incorporating an additional $\sim 260000$ scenes, the current version is the second data product after resolution improvement and water body coverage refinement, with a resolution of 1 arc second $(\sim 30 \mathrm{~m})$ referenced to the WGS84/EGM96 geoid. The ASTER GDEM v2 data were distributed as $227021^{\circ} \times 1^{\circ}$ tiles. The dataset covers a nearly global geographic extent, with an accuracy of $17 \mathrm{~m}$ at the $95 \%$ confidence level (Tachikawa et al. 2011). However, it is known to be influenced by a variety of artifacts and anomalies that limit its use without any further preprocessing. With the elevation measured from the Earth's reflective surface, the accuracy of the ASTER GDEM v2 is sensitive to the land-cover type, such as forest canopy or buildings (Arefi and Reinartz 2011).

A comparison of the three datasets is shown in Fig. 1. The sample data are from a small 
area (elevation from 614 to $1815 \mathrm{~m}$ ) with relatively rugged terrain in Sichuan province, China. It can be seen that the SRTM-3 data cannot clearly reflect the details of the terrain surface, while the ASTER GDEM is significantly affected by noise. Moreover, the SRTM data show obvious advantages in delineating topographic feature lines, compared with the ASTER data.

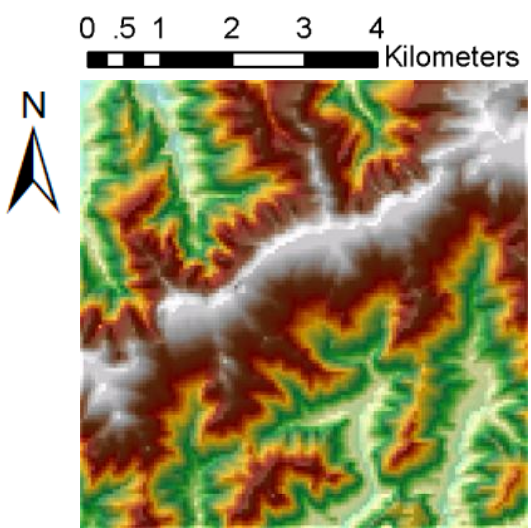

(a)

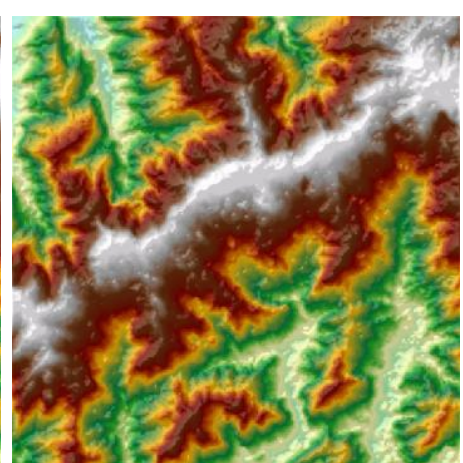

(b)

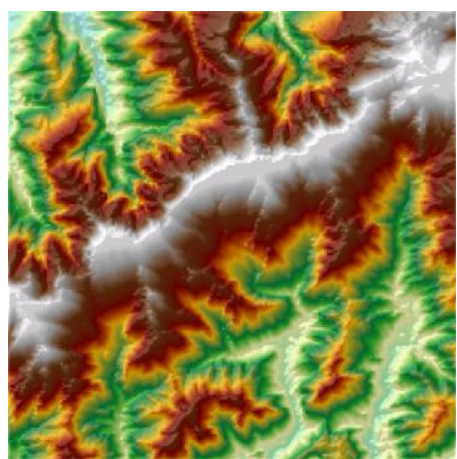

(c)

Fig. 1. A comparison of the three global DEM datasets in a sample area (elev: $\sim 614-1815 \mathrm{~m}$ ). From left to right: 90-m SRTM-3, 30-m ASTER GDEM v2, and 30-m SRTM-1 data, respectively.

\subsection{ICESat GLAS}

The GLAS system was the laser scanning instrument carried onboard NASA's ICESat satellite launched in January 2003. The ICESat science mission started in 2003, and ended due to the failure of its instrument seven years later (Satgé et al. 2015). The primary objective of the GLAS instrument was to measure ice sheet elevation and elevation changes over time. The secondary products included measurements of cloud and aerosol height profiles, and land elevation and vegetation cover (http://nsidc.org/data/icesat). In this paper, we utilize the GLAS/ICESat L2 Global Land Surface Altimetry Data Version 34 (GLAH 14) for the DEM accuracy enhancement and assessment. The GLAS instrument produced laser spots on the Earth's surface with a 70-m diameter. Fig. 2 shows the spatial extent of the 
ICESat tracks collected in March 2003, where it can be seen that the data points distribute over the entire land surface of the Earth. The spacing between the along-track points is nearly $170 \mathrm{~m}$, while the cross-track spacing varies from several kilometers to several tens of kilometers, based on the latitude (Abshire et al. 2005).

The vertical accuracy of the GLAS points can be better than $1 \mathrm{~m}$ (Zhang et al. 2011). However, outliers caused by poor acquisition conditions, such as cloud reflections and saturated waveforms, need to be filtered and the accuracy needs to be refined before using the ICESat elevation products (Huber et al. 2009; Arefi and Reinartz 2011). The GLA14 data provide the centroid height of the return echo, and offer parameters related to the return pulse characteristics and the measurement conditions (NSIDC 2014). Different criteria have been proposed to select ICESat samples satisfying the accuracy demand in various applications (Huber et al. 2009; González et al. 2010). The principal parameters of the GLAS data include the number of Gaussian peaks, the surface slope and roughness, the cloud layers, the pulse energy, and the surface type (Arefi and Reinartz 2011). Furthermore, the reference geoid needs to be converted to be consistent with the SRTM and ASTER data, from TOPEX/Poseidon to WGS/EGM96 (Baghdadi et al. 2011). The specific processing flow is explained in Section 3.1.1. 


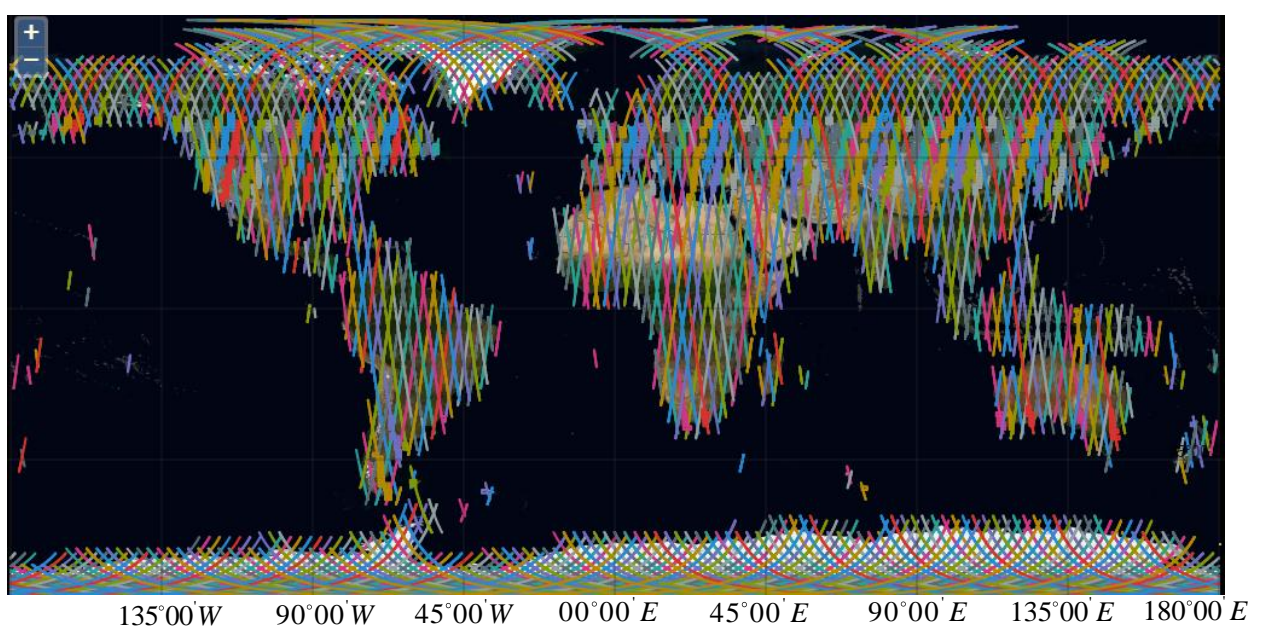

Fig. 2. The spatial extent of the ICESat tracks collected in March 2003.

\section{Processing flow}

The general processing flow of the proposed approach is shown in Fig. 3, and consists of the following steps. Firstly, for each DEM tile $\left(1^{\circ} \times 1^{\circ}\right)$ with voids in the SRTM-1 data, the accuracy of the ASTER GDEM is enhanced using the high-accuracy ICESat data. The accuracy enhancement process is performed with an ANN model. After the accuracy enhancement, the SRTM voids are filled with the corresponding corrected ASTER GDEM values. The triangular irregular network (TIN)-based delta surface fill (DSF) method (Luedeling et al. 2007) is then utilized to remove the vertical bias between the SRTM data and the corrected ASTER GDEM surfaces. Finally, postprocessing is performed with an outlier filter applied to all the data tiles, including the non-void tiles. All these processing steps contribute to a continuous global DEM product with improved quality. The specific details of the processing flow are described in the following. 


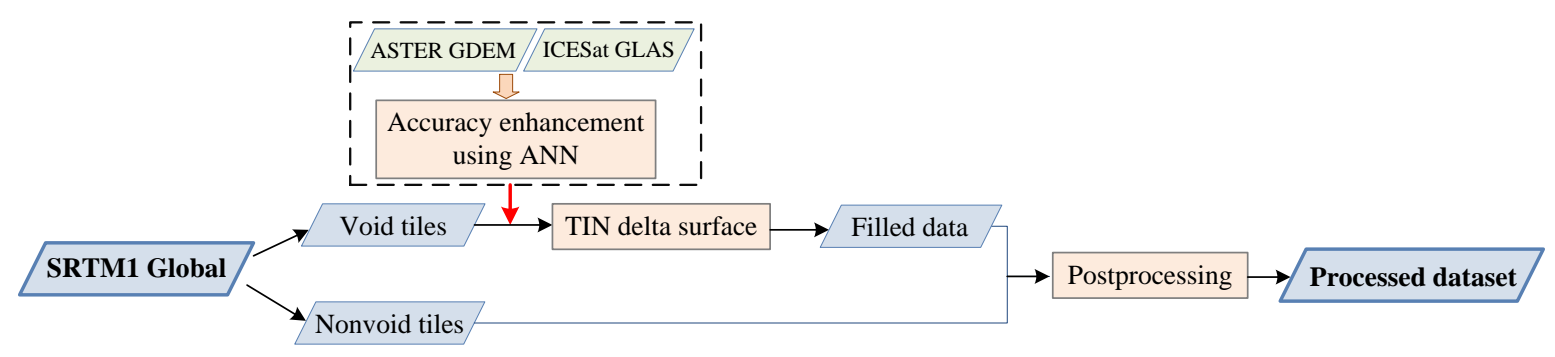

Fig. 3. The flow chart of the whole framework.

\subsection{Accuracy enhancement of the ASTER GDEM using ICESat GLAS data}

\subsubsection{Processing of the ICESat GLAS data}

Before the accuracy enhancement, the ICESat GLAS data first need to be preprocessed. ICESat elevations correspond to the ellipsoidal heights with reference to the TOPEX ellipsoid. To fuse the SRTM-1 and ASTER GDEM data, the reference datum needs to be converted to the WGS84 geographical coordinate system and the EGM 96 geoid model. The difference in geodetic latitude and longitude produces a horizontal displacement of less than a meter, which can be ignored. The vertical datum conversion from the TOPEX ellipsoid to the WGS84 ellipsoid can be approximated by $h_{\text {WGS } 84}=h_{\text {Topex }}-0.707 m$ (Baghdadi et al. 2011). The ellipsoidal elevations $\left(h_{W G S 84}\right)$ are then converted to orthometric elevations $H=h_{W G S 84}-N$, where $N$ is the EGM96 geoid value (Bhang et al. 2007).

After the datum conversion, the GLAS points need to be filtered to exclude the low-quality outliers. Theoretically, the outliers can be detected with the provided parameters referring to the data structure (González et al. 2010). In this paper, our main target is to select the ICESat laser points whose accuracy meets the SRTM requirements. The outliers with gross errors caused by cloud reflections and atmospheric noise during the time of data acquisition are the main concerns. The commonly used criteria for erroneous 
points filtering are considered, including the number of peaks, the signal width and the received energy (Huber et al. 2009; Arefi and Reinartz 2011). Specifically, the 6-peak points are removed to eliminate most forest points. Moreover, the points with received energy lower than $10 \mathrm{fJ}$ and a signal width of less than $25 \mathrm{~m}$ are selected. In addition, a threshold for the height difference between the ICESat values and the corresponding reference DEM datasets is also set to filter the ICESat measurements. The ICESat points are excluded from the analysis if the difference between the ICESat data and both the ASTER GDEM and SRTM data exceeds $200 \mathrm{~m}$.

\subsubsection{Accuracy enhancement using the ANN model}

An ANN can be a powerful tool to derive meaning from complicated datasets, no matter how the errors are distributed or what the relationship is between the data (Hsu et al. 1995; Luk et al. 2000; Öztopal 2006). With the help of the ANN's remarkable ability to learn the statistical information from a training dataset composed of the corresponding ASTER GDEM and ICESat GLAS data, the ASTER GDEM values with a similar pattern to the training samples can be corrected accordingly.

The structure of a feed-forward ANN is shown in Fig. 4. The network usually consists of a number of neurons connected by links through several layers. It can be characterized as parallel interconnections of cross-layer neurons, which link the input and output data through a set of weighted transfer functions. By creating a network with $r$ input neurons, $s$ hidden neurons, and $p$ output neurons, the corresponding variables for the input training samples are defined as $\boldsymbol{x}=\left(x_{1}, \cdots, x_{r}\right), \boldsymbol{h}=\left(h_{1}, \cdots, h_{s}\right)$, and $\boldsymbol{o}=\left(o_{1}, \cdots, o_{p}\right)$. The output for each layer can be calculated as: 


$$
h_{j}=f_{1}\left(\sum_{i=1}^{r} x_{i} w_{i j}^{h}+\beta_{j}\right) \quad o_{k}=f_{2}\left(\sum_{j=1}^{s} h_{j} w_{j k}^{o}+\tau_{k}\right)
$$

where $w_{i j}^{h}$ indicates the weight for the link from the input neuron $x_{i}$ to the hidden neuron $h_{j}$, and $w_{j k}^{o}$ is the weight for neurons $h_{j}$ and $o_{k} \cdot \beta_{j}$ and $\tau_{k}$ represent the bias for neurons $h_{j}$ and $o_{k}$, respectively. In the above equation, $f_{1}(\cdot)$ and $f_{2}(\cdot)$ indicate the transfer functions, which are used to simulate the relationship between the connections. The transfer functions are continuous and bounded, and are usually chosen from linear, logistic, and sigmoid functions. The error function obtained using the actual output and the ideal output can be minimized by adjusting the weight matrix. A back-propagation (BP) algorithm is usually used for the optimization problem, with the output errors propagated backwards through the network, and changes are made to the weights in each layer to reduce the error signal (Zhang et al. 2008). Each weight is updated in the nth iteration as:

$$
\begin{aligned}
& w_{j k}^{o n+1}=w_{j k}^{o n}-\eta \frac{\partial E}{\partial w_{j k}^{o}} \\
& w_{i j}^{h n+1}=w_{i j}^{h n}-\eta \frac{\partial E}{\partial w_{i j}^{h}}
\end{aligned}
$$

In this equation, $E=\sum_{k=1}^{p}\left(y_{k}-o_{k}\right)^{2}$ is the error function calculated with the target vector $\boldsymbol{y}$ and actual output $\boldsymbol{0}$. The changes of the weight are determined by the learning rate $\eta$, which is a parameter that influences the speed and quality of the learning. A higher learning rate causes faster iteration and more accurate training; however, this sometimes leads to overfitting in the training process. 


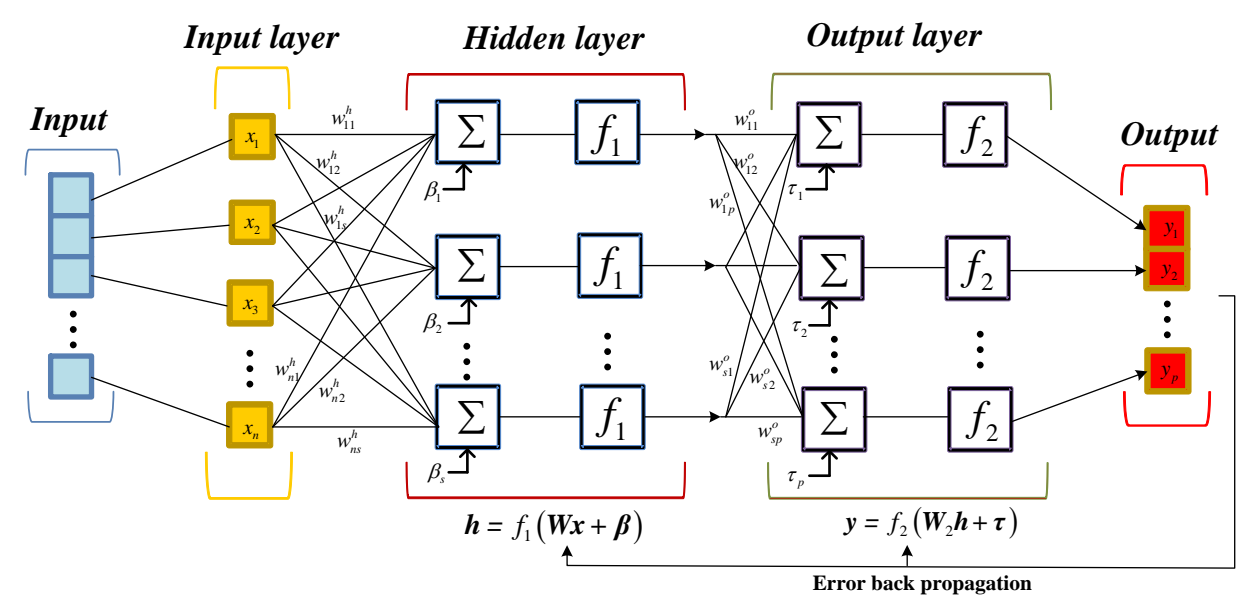

Fig. 4. The structure of a feed-forward ANN. The expected output is obtained with signal feed-forward propagation, while the weight is adjusted iteratively through error back-propagation.

Specifically, in our case, the ICESat data collected from 2003 to 2008 are used as the training dataset, while the data points in 2009 are used for the result validation after the accuracy correction. For each ASTER tile, we extract the neighboring patch of every pixel where an ICESat point is located. The extracted patch is then converted to an input vector corresponding to the target ICESat value. Through traversing every ICESat point, we can obtain the input matrix, whose columns are composed of the extracted input vectors. The ICESat data points are used to construct the target vector, as shown in Fig. 5.
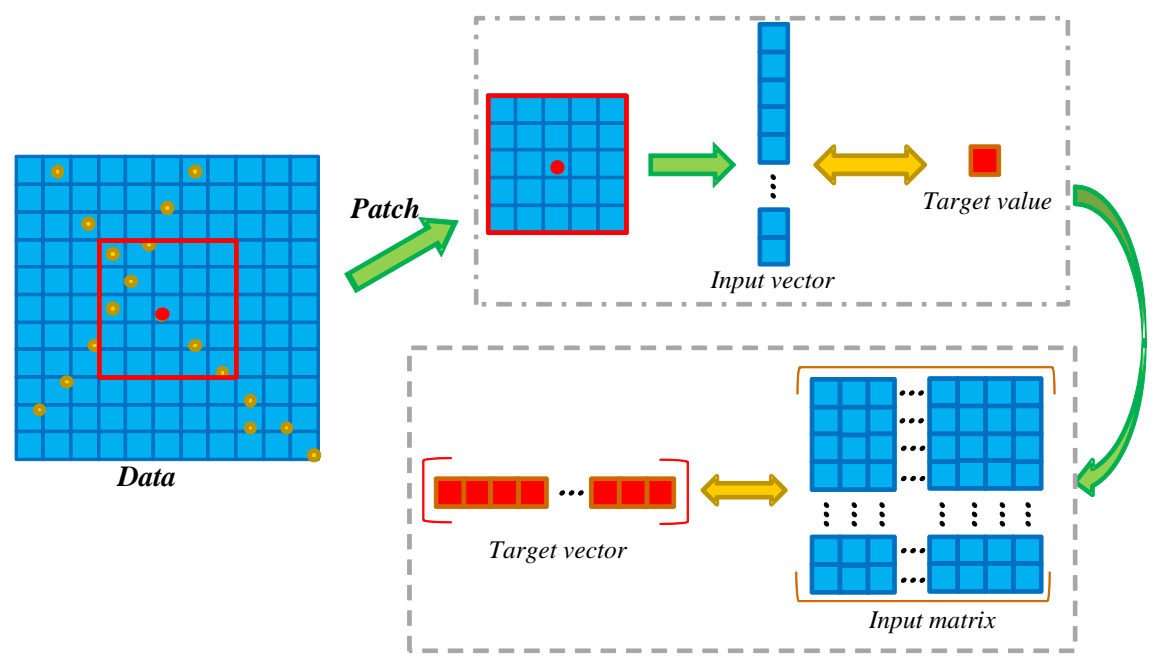

Fig. 5. Training sample construction for the ANN model.

In addition to the ASTER values, we also incorporate slope information and the spatial 
location (latitude and longitude coordinates) to construct the training dataset. The slope information is related to the varying terrain relief. Generally, the anomalies occur more often in the rugged terrain with large slope. However, not all the data points within the high-slope relief are contaminated with large errors (Li et al. 2013). To avoid introducing errors to the normal values, spatial information is included in the model. The neighboring coordinates can reflect the similar spatial pattern, thus taking the influence of spatial variation into account. The combination of terrain variation and spatial information can help us to learn the relationship between the ASTER GDEM values and the control points. Fig. 6 shows the ANN enhancement model. A supervised BP neural network is created to learn the approximated representations of the input ASTER GDEM data to the high-accuracy ICESat values from the normalized training set. After the training process, the optimal weight matrix can be obtained, and thus other pixels without ICESat points located can also be mapped based on the learned representations as the test data.

There are several parameters in the ANN enhancement model, including the neurons for each layer, the transfer function, the learning rate, etc. Among them, the network structure and the learning rate are the most sensitive parameters. In our experiments, we found that a network with six neurons for the first layer and four neurons for the second layer is suitable for the correction process (the neurons of the output layer are determined by the dimension of the target vector), while the "purelin" function is adopted for the transfer function. The learning rate is set as 0.001 . 


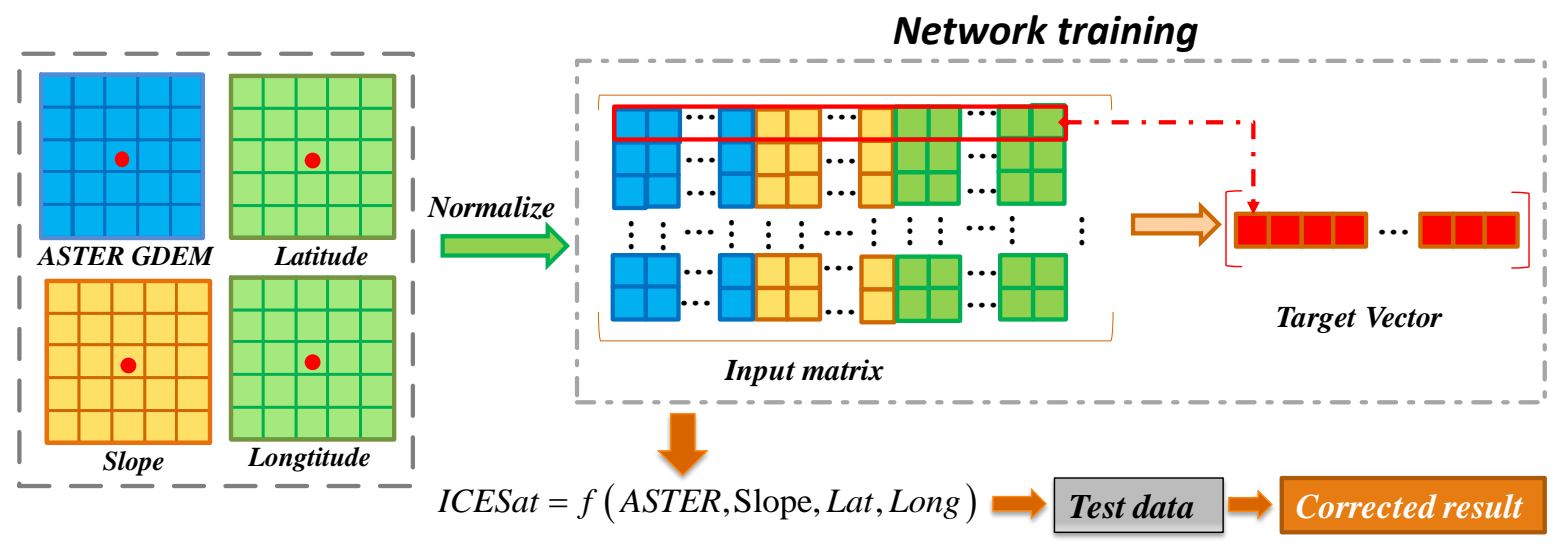

Fig. 6. The ANN model of accuracy enhancement for the ASTER GDEM.

\subsubsection{Model performance validation}

During the training process, the network randomly divides the training dataset into three parts, which are respectively used for training, validation, and testing. The validation set is mainly used to minimize overfitting by tuning the parameters during the training process, while the test set plays the role of assessing the performance of the trained network (Ripley 2007). However, considering the zonal distribution of the ICESat points, the random divided sets with neighboring spatial locations still cannot easily reflect the real predictive ability of the ANN model. Therefore, we further validate the model performance by manually separating the reference data points into two parts for training and testing, respectively.

An example is given in Fig. 7. In Fig. 7, all the points within the rectangular area are excluded from the training process and are used for the independent testing. Hereafter, they are called the "independent test dataset". The remaining points outside the rectangle, which are referred as the "training dataset", are input into the ANN model. The assessment results are given in Table 1, where the root-mean-square error (RMSE) values of both the training 
and independent test datasets are calculated. For a better comparison and analysis, the results of the original ASTER data, multiple linear regression (MLR) and the ANN method using different amounts of training data are also presented. The percentage numbers in the brackets indicate the training dataset proportion. For example, in the case of " $10 \% ", 10 \%$ of the points in the training dataset are randomly chosen for training, while the other $90 \%$ are used for the validation and test set in the ANN model. In the case of the MLR model, all the points in the training dataset are involved in the regression process.

It can be seen from the table that the test accuracy of the MLR result shows a weak predictive ability of the MLR model. With sufficient training samples, the ANN model performs better than the MLR method. There is an improvement in the ANN results as the training samples increase, which is mainly reflected in the independent test accuracy. The quantitative results become stable when $50 \%$ of the training points are involved in the training process. Given the quite different spatial coverage of the training and independent test datasets, the test results can provide us with some more reliable information about the predictive ability of the model.

In addition to the sample case, a great variety of experiments are also conducted to validate the model performance. The experimental results confirm that the simulation process performs well. In the tiles with sufficient ICESat points, the assessment results using the ICESat training dataset and the independent test dataset are comparative, which indicates that the neural network is capable of predicting the relationship between the DEM data and the target laser points through learning from the training set. 


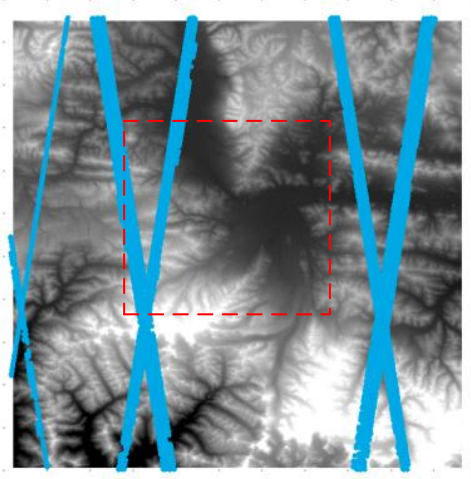

Fig. 7 The distribution of the ICESat data over data tile numbered 'N28E086'. The 3788 points within the rectangle area are excluded from the training process and are used for the independent test validation, while the remaining 15845 points are used as the training dataset.

Table 1 The validation results of the training process.

\begin{tabular}{ccccccc}
\hline \hline Unit: $m$ & ASTER & MLR & ANN (1\%) & ANN (10\%) & ANN (50\%) & ANN (70\%) \\
\hline RMSE_train & 23.753 & 22.840 & 21.934 & 20.746 & 20.593 & 20.579 \\
\hline RMSE_test & 23.225 & 23.928 & 24.892 & 22.339 & 22.100 & 22.031 \\
\hline \hline
\end{tabular}

\subsection{Void filling of the SRTM-1 data and delta surface calculation}

Considering the error distribution of the DEM datasets and the spatial coverage of the ICESat data, only the void regions with a relatively low accuracy (and the bordering pixels) are corrected using ICESat points. After the accuracy enhancement, the voids of the SRTM-1 data can be filled with the corresponding corrected ASTER GDEM values.

The crux of the void-filling problem is a reliable source of auxiliary data and the method used to eliminate the bias between the datasets (Ling et al. 2007; Karkee et al. 2008). The main methods used to deal with the vertical bias in multi-source DEM fusion include fill and feather (FF) (Dowding et al. 2004), DSF (Grohman et al. 2006), and TIN-DSF (Luedeling et al. 2007). The FF algorithm simply uses a constant to approximate the bias, which ignores the varying terrain surface within the void regions. Comparatively, the DSF 
method uses the non-void values in the surface to compute the difference surface between the input data, referring to a "delta surface". The voids in the delta surface are then filled using a geostatistical interpolation method, considering the local surface trend (Grohman et al. 2006). However, this method is only optimal for small voids. A more promising method is the TIN-based DSF method, which quantifies the pixel-specific difference between the surfaces based on the TIN base surface (Luedeling et al. 2007).

Among these methods, TIN-DSF can deal with the variable terrain surface within the voids by utilizing the available height information, thus obtaining more promising results. Following the idea of the TIN-DSF method (Luedeling et al. 2007) (Fig. 8), the SRTM surface is merged with the corrected ASTER GDEM data in this paper. The DSF method computes an adjustment of the fill source to the SRTM. As no SRTM data information is provided in the void regions, the neighboring valid elevations are incorporated to estimate the delta surface. The detailed steps of the method are as follows. Firstly, we create two base surfaces using the points bordering the voids from both the SRTM data and the corrected ASTER GDEM data with the natural neighbor interpolation method, as shown in Fig. 8(c). The bordering pixels are extracted in a buffer zone of five pixels around the data voids. The delta surface between the ASTER surface and the ASTER base surface can then be obtained. Assuming that the surface morphology of the ASTER and SRTM data are basically consistent within the region, the relative elevation delta surface for the ASTER DEM can be applied to the SRTM model. To avoid any abrupt changes in the elevation differences and the errors brought about by the interpolation method, the delta surface is then filtered to obtain a relatively smooth surface. Finally, a seamless merged elevation 
model can be acquired by adding the delta surface to the SRTM base surface as:

$$
S_{C}=S_{b}+A_{C}-A_{b}
$$

where $S_{b}$ and $A_{b}$ indicate the base surface of the SRTM and ASTER data, respectively. $A_{C}$ is the corrected ASTER values obtained within the void regions. The whole process is also illustrated in Fig. 8. Considering that some pixels bordering the voids are contaminated with noise, a slight feathering is performed to smooth the transitional regions.
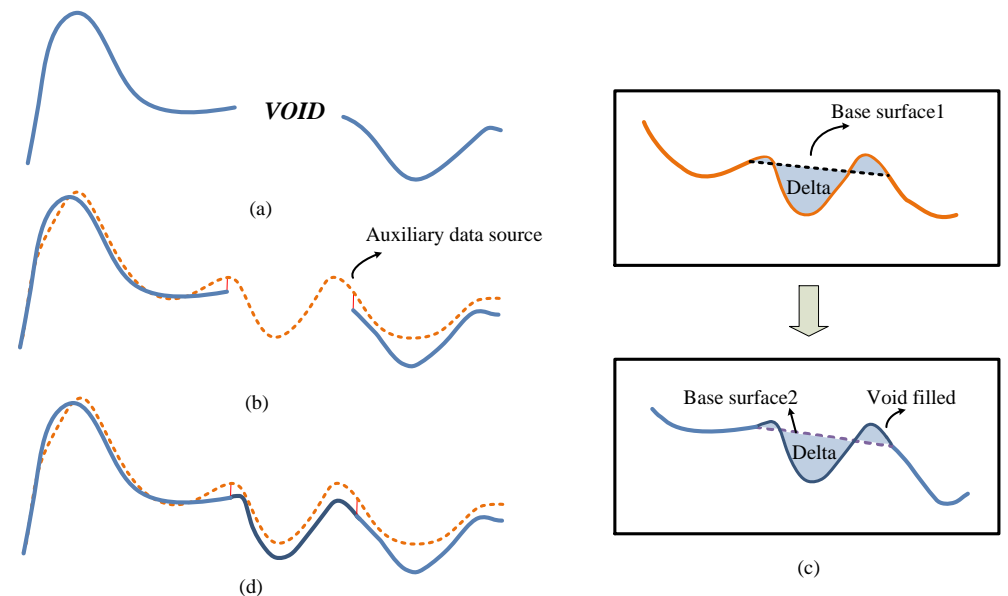

(c)

Fig. 8. An illustration of the process of delta surface calculation. (a) represents a projected DEM surface with voids, while (b) indicates that the auxiliary data source for void filling might be mismatched with the original surface due to the varying vertical bias, and (c) illustrates the process of creating a delta surface with the TIN base surface. A comparison of the original filling source and the filled result using the TIN-DSF method is shown in (d).

\subsection{Postprocessing}

After all the tiles with voids have been filled following the above process, we post-process all the tiles using an adaptive outlier filtering algorithm. This helps to reduce the noise and outliers in the corrected DEM data. For every pixel, a neighborhood patch $(5 \times 5)$ surrounding the central pixel is extracted. Through calculating the difference between the neighboring pixels and the central pixel, the number of pixels whose difference is greater than twice the standard deviation can be counted. If $75 \%$ of the elevation difference values 
within the patch are counted as above the threshold, or the height value is clearly abnormal (e.g. above $8850 \mathrm{~m}$ ), the central pixel is classified as an outlier. The corresponding pixel is filtered by averaging the neighboring pixels. This algorithm is simple to implement and can avoid excessive smoothing of the detailed terrain information.

\section{Results and analysis}

The final processed dataset comprises seamless SRTM-1 land elevation data covering $60^{\circ} \mathrm{N}$ to $\sim 56^{\circ} S$ globally. In this paper, the accuracy of the processed dataset within China is analyzed in detail, while the ASTER GDEM v2 and the SRTM-3 data are included in the comparison. For a more detailed and comprehensive accuracy analysis, all the valid ICESat laser points collected from 2003 to 2009 are used as reference data for the validation.

\subsection{Study area}

The landscape of China is vast and diverse, ranging from coastal plains to high-altitude plateaus, covering over 9.6 million square kilometers. In Fig. 9, most of the land area of China is shown (except the sea areas and some small islands), where it can be observed that the elevation basically increases from the eastern coastal area to the western mountainous area.

The voids in the original SRTM-1 global data are shown in Fig. 9(a). We can see that the voids mainly occur in the western part of China, where the high elevation and varying slopes result in rugged terrain. Specifically, there are 85 tiles with voids in China, which are mainly distributed in the Tibet Plateau, the Hengduan Mountains, and the edge of the basins in the Xinjiang Uygur Autonomous Region. Among the tiles, three are contaminated with 
over $30 \%$ void pixels. The total number of void regions within China is 172503 , which are composed of 95255394 void pixels in total. Among them, $7.9 \%$ of the voids are small with less than 20 pixels, while $20.7 \%$ of the voids are relatively large with over 200 pixels. Void filling and accuracy improvement was conducted for the SRTM-1 data tiles in China by incorporating the ASTER GDEM and ICESat GLAS data, following the processing flow described in Section 3. A color map of the seamless data after processing is shown in Fig. 9(b). In the next sections, we present both the visual performance and the vertical accuracy assessment of the processed data. Two sample areas (the highlighted regions in Fig. 9(b)) are chosen for a detailed analysis. Furthermore, a channel network extracted from the datasets is also given for assessment.

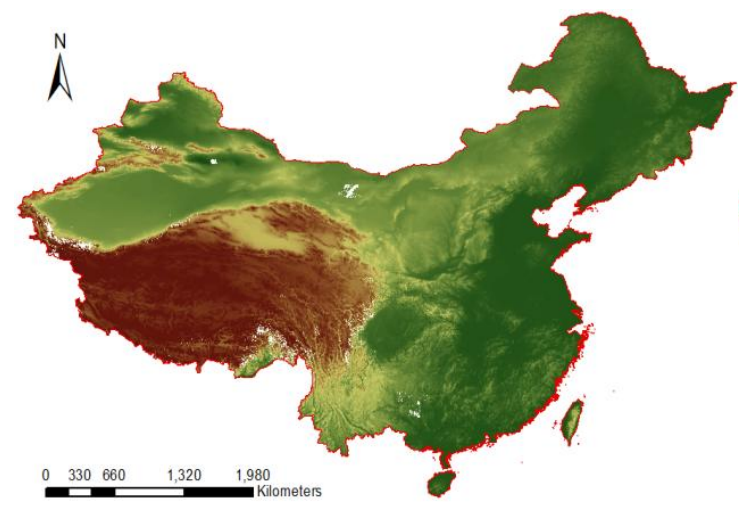

(a)

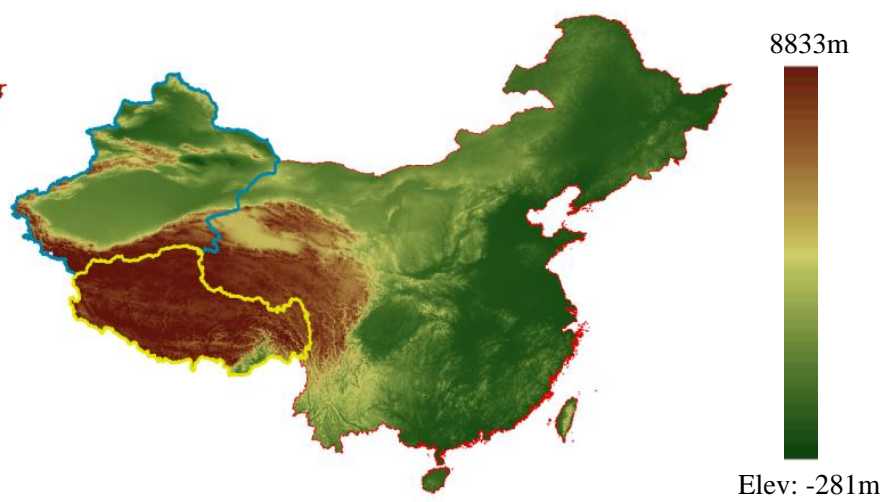

(b)

Fig. 9. Color map of the topography of most of the China land surface. (a) represents the elevation map of SRTM-1 with voids, while (b) is the processed dataset. The highlighted parts in (d) are the study areas for the accuracy assessment: the Tibet Autonomous Region (yellow) and the Xinjiang Uygur Autonomous Region (blue).

\subsection{Vertical accuracy assessment and analysis}

The assessment of the processed data was performed over the whole of China and within two sample regions in China: the Tibet Autonomous Region and the Xinjiang Uygur Autonomous Region. Both of these regions are located in the western part of the Chinese 
mainland, where data voids are common due to the complex terrain. Firstly, the overall accuracy comparison of the global DEM products in China is given, followed by the detailed analysis of the processed dataset in the Tibet and Xinjiang regions. Three indexes are employed for the vertical accuracy: the mean absolute error (MAE), the standard deviation (SD), and the RMSE. We denote $d$ as the elevation error calculated by the difference between the corresponding DEM value and the reference data, and $n$ as the number of validation samples, so:

$$
M A E=\sum_{i=1}^{n}\left|d_{i}\right| / n, S D=\sqrt{\frac{\sum_{i=1}^{n}\left(d_{i}-\bar{d}\right)^{2}}{n}}, R M S E=\sqrt{\sum_{i=1}^{n} d_{i}^{2} / n}
$$

where $\bar{d}$ is the mean value of the error vector. Compared with the MAE, the RMSE can reflect the error distribution more precisely, especially where there are gross errors.

\subsubsection{Overall accuracy assessment and discussion}

We give the results of the overall accuracy validation of the DEM datasets covering most of China (Fig. 8), shown in Tables 1-2. In China, over 15.27 million ICESat points were used for the accuracy validation, while approximately 53000 points were distributed within the voids. The accuracy statistics for the void and non-void regions are given separately in Table 1. In addition to the processed data, the validation results of the ASTER GDEM v2 and the SRTM-3 data are also given for comparison. The no-data regions in the earlier releases of the SRTM-3 data were filled following the method described by Reuter et al. (2007). The small holes were filled by the use of interpolation algorithms, and then the auxiliary DEMs were used for the large voids, with cleaning of the surface to reduce pits and peaks. However, we previously found that even when the voids in the SRTM-3 v4.1 
data had been filled, the accuracy of the void regions was still unreliable (Yue et al. 2015).

The accuracy in the void areas is clearly affected by the anomalies and errors, which are mainly brought about by the steep slopes and rugged terrain. The accuracy of the SRTM-3 data is much lower than the ASTER GDEM data within the void regions, which is probably due to the unreliable void-filling results in the earlier release. After correction, the elevation values are more accurate in the rugged terrain. Excluding the voids, the vertical accuracy is much better and more stable. There is little difference between the statistics for the SRTM-1 data and the processed dataset for the non-void areas, because only a few outliers were filtered in these regions to avoid introducing unnecessary errors. Given this fact, only the results for the processed dataset are given here. The MAE of the SRTM-3 data is slightly less than the MAE of the ASTER GDEM data within the non-void areas, while the SD and RMSE values behave in the opposite way. This indicates that the accuracy of the SRTM-3 data is more affected by gross errors, which might be due to the fact that there are more voids in the unfilled SRTM-3 data than the newly released SRTM-1 data. The errors introduced in the void-filling process for the SRTM-3 data were not eliminated by excluding the void regions in the SRTM-1 data. In Table 2, we compare the overall accuracies, where the overall accuracy of the SRTM-1 data was calculated with the voids filled directly using the corresponding ASTER values. The improvement is clearly reflected in the RMSE values. The overall accuracy of the processed dataset obtained in this study in China is about $15.2 \mathrm{~m}$. However, the accuracy varies from region to region based on the terrain complexity. As expected, the accuracy of the western areas with more rugged terrain is below the overall average level, while the eastern flat terrain has a relatively higher 
accuracy.

Table 1 The accuracy statistics in China for the void and non-void areas.

\begin{tabular}{cccc|ccc}
\hline \hline \multirow{2}{*}{ Unit: (m) } & \multicolumn{3}{c|}{ Void } & \multicolumn{3}{c}{ Non-void } \\
\cline { 2 - 7 } & ASTER & SRTM-3 & Processed & ASTER & SRTM-3 & Processed \\
\hline MAE & 35.088 & 100.160 & 25.038 & 10.816 & 9.495 & 5.677 \\
\hline SD & 119.320 & 183.690 & 54.380 & 18.960 & 19.804 & 14.563 \\
\hline RMSE & 121.140 & 191.549 & 55.160 & 19.182 & 20.007 & 14.853 \\
\hline \hline
\end{tabular}

Table 2 The overall accuracy statistics in China.

\begin{tabular}{ccccc}
\hline \hline \multirow{2}{*}{ Unit: $(\mathrm{m})$} & \multicolumn{4}{c}{ Overall } \\
\cline { 2 - 5 } & ASTER & SRTM-3 & SRTM-1 & Processed \\
\hline MAE & 10.905 & 9.814 & 5.784 & 5.749 \\
\hline SD & 20.249 & 22.809 & 16.215 & 14.918 \\
\hline RMSE & 20.467 & 22.963 & 16.487 & 15.205 \\
\hline \hline
\end{tabular}

\subsubsection{Tibet Autonomous Region}

The Tibet Autonomous Region is located in the southwestern part of China, spanning over 1.2 million square kilometers. It covers part of the Tibet Plateau, and is representative of harsh and rugged terrain. The southern part of the Tibet Autonomous Region is bounded by the Himalayas, while to the north there is a broad mountainous area. The Tibet Plateau is known as "the roof of the world", and this region is the highest region on Earth (Ma et al. 2008). In the northern part, the elevations reach an average of over $4500 \mathrm{~m}$. The voids of the SRTM-1 data within China are distributed mainly in this region (Fig. 10), where 31 $1^{\circ} \times 1^{\circ}$ data tiles containing 72285 void areas exist. There are 2.29 million valid ICESat data points over this region, with about 14000 points distributed within the voids. 


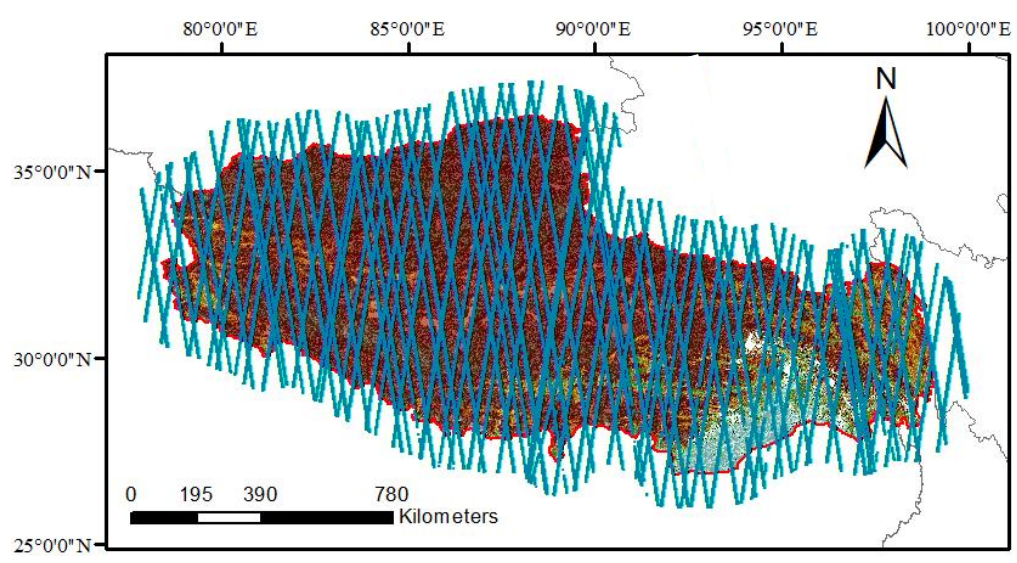

Fig. 10. The shaded relief map of the SRTM-1 data in the Tibet Autonomous Region (elev: $-1-8833 \mathrm{~m}$ ). The voids mainly occur in the southern part, and are shown as white pixels. The blue tracks indicate the spatial coverage of the ICESat data points.

After void filling and accuracy improvement, the accuracy validation results of the datasets are shown in Table 3, where we present both the accuracy of the void areas and the overall region. As in the previous analysis, the accuracy of the DEMs is significantly affected by the steep slopes, where voids are common. Although the voids in the ASTER GDEM and the SRTM-3 products in the rugged terrain have been filled, the datasets are still far from being suitable for direct use without further processing. The MAE and RMSE values indicate that the ASTER data might be more significantly affected by anomalies or incorrect height values in this region.

After correction using the ICESat laser points with more accurate elevation information, the accuracies of the void areas show an obvious improvement, with the number of outliers reduced, compared with the original data. However, the errors cannot be eliminated completely due to the limited accuracy of the original data, as well as the influence of the complex topography. The overall accuracy is also validated in this region. The improvement in MAE is relatively slight due to the small proportion of void pixels 
compared to the total number of pixels, while the RMSE value shows that the large errors are corrected and the accuracy shows a significant improvement.

Table 3 The accuracy statistics in the Tibet Autonomous Region for void pixels and the overall region, respectively.

\begin{tabular}{cccc|cccc}
\hline \hline \multirow{2}{*}{ Unit: $\mathrm{m}$} & \multicolumn{3}{c|}{ Void } & \multicolumn{4}{c}{ Overall } \\
\cline { 2 - 8 } & ASTER & SRTM-3 & Processed & ASTER & SRTM-3 & SRTM-1 & Processed \\
\hline MAE & 64.942 & 70.048 & 38.118 & 14.984 & 14.612 & 7.798 & 7.619 \\
\hline SD & 214.275 & 120.499 & 74.397 & 27.470 & 25.046 & 20.150 & 11.251 \\
\hline RMSE & 218.326 & 120.697 & 76.349 & 28.032 & 25.687 & 21.127 & 12.838 \\
\hline \hline
\end{tabular}

The statistical information for each data tile is also presented. In Fig. 11, the red curve shows the ratio of void pixels in each tile $(3601 \times 3601)$, and the blue curve represents the number of ICESat points distributed within the voids. Among the 31 tiles in the Tibet Autonomous Region, there were five tiles where less than 50 ICESat points were distributed within the voids, or the ratio of void pixels was less than $0.05 \%$. Therefore, the accuracy curves of the other 26 tiles are shown in Fig. 12 to avoid a biased conclusion due to insufficient data samples. It can be seen from the figure that the curves of the SD and RMSE show the similar trends, and the improvement of the processed result compared to the original data varies with the topographic relief, the size and distribution of the voids, and the density of the ICESat points. In Fig. 13, a sample area within the tile numbered 'N28E086' is given for a visual inspection. In this tile, $4.49 \%$ of the pixels were voids, and 20465 ICESat points were included in the accuracy improvement. Using the corrected ASTER values, the voids in the SRTM data were filled, and the vertical bias was dealt with using the TIN-DSF method. The processed dataset can then be used as a seamless elevation product. 


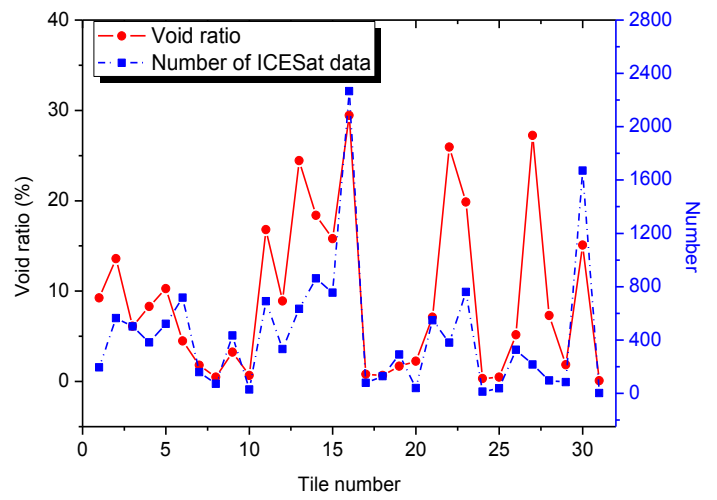

Fig. 11. Distribution of voids and ICESat points distributed within the voids in the void tiles in the Tibet Autonomous Region.

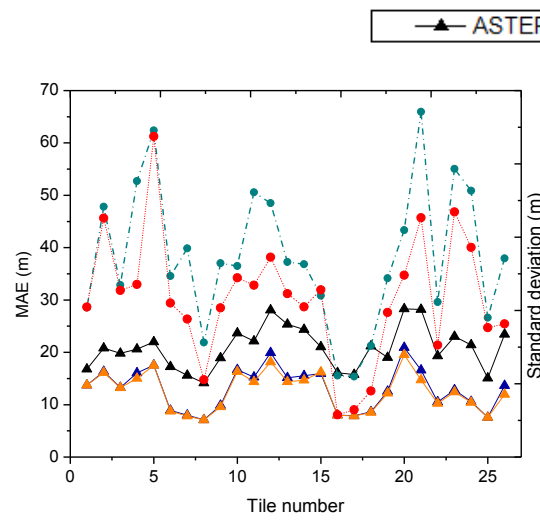

(a)

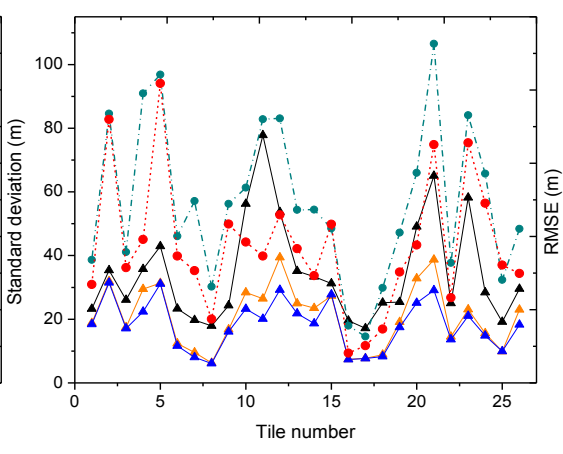

(b)

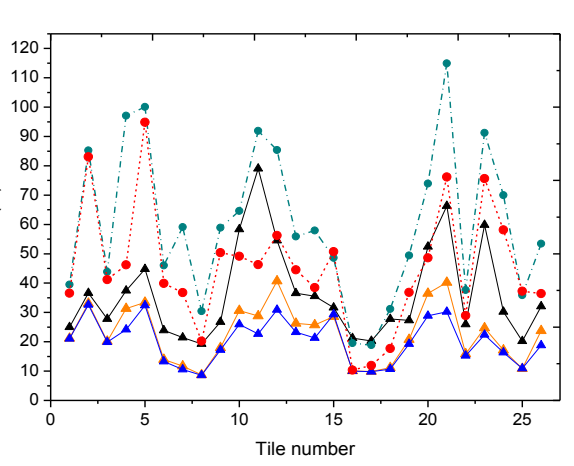

(c)

Fig. 12. The vertical accuracy assessment of the void data in the Tibet Autonomous Region. (a)-(c) indicate the MAE, SD, and RMSE values for the 26 tiles with voids, respectively.

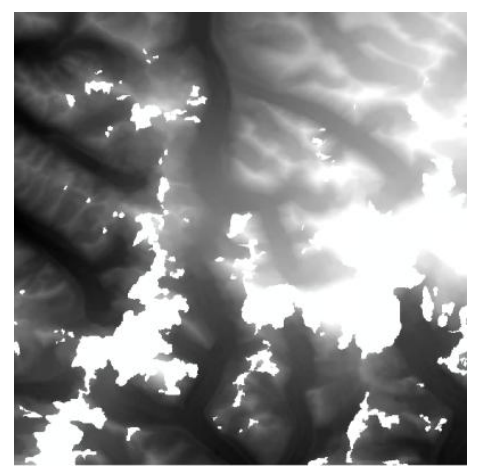

(a)

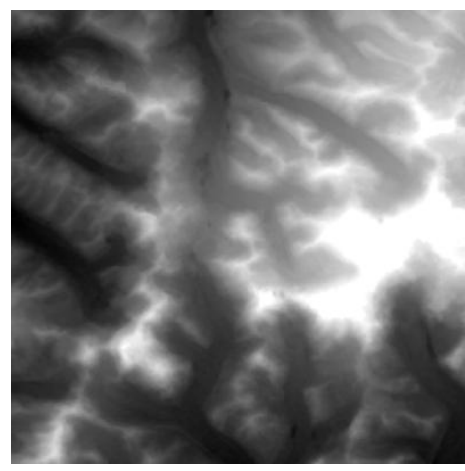

(b)

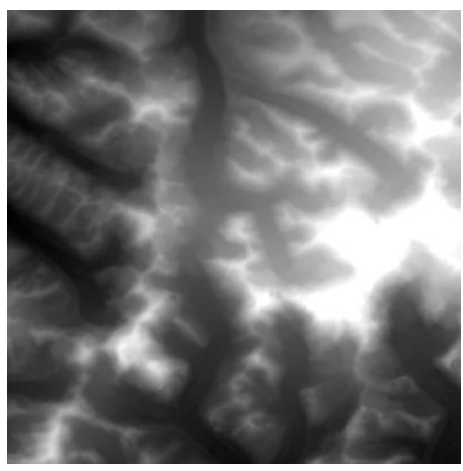

(c)

Fig. 13. A sample area from the Tibet Autonomous Region. (a)-(c) indicate the original SRTM-1 data with voids, the ASTER GDEM data for void filling, and the processed result, respectively.

\subsubsection{Xinjiang Uygur Autonomous Region}

The second sample area we chose to analyze in this paper is the Xinjiang Uygur 
Autonomous Region, which is located in the northwest of the country, bordering the Tibet Autonomous Region. It is the largest Chinese administrative division, spanning over 1.6 million kilometers. As mentioned before, the voids in the original data are distributed mainly in the western part of China. In the Xinjiang Uygur Autonomous Region, there are 26 tiles containing voids, composed of 41613 void areas. It can be seen from Fig. 14 that most of the voids are on the southern edge of this region. This is because the extremely rugged Karakoram, Kunlun, and Tianshan mountains occupy much of Xinjiang's borders. The central part of the Xinjiang Uygur Autonomous Region, which mainly consists of desert landscapes, is divided into two large basins by the Tianshan mountain range (Yang et al. 2002; Jia et al. 2004). In this region, there are over 3.84 million ICESat points for the accuracy validation, with 20709 reference points distributed within the voids.

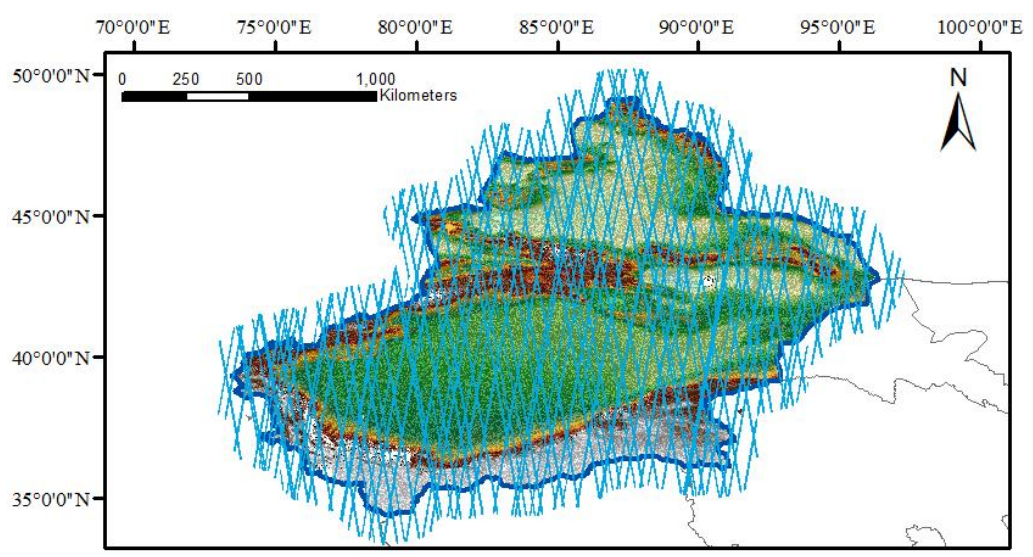

Fig. 14. The shaded relief map of the SRTM-1 data in the Xinjiang Uygur Autonomous Region (elev: $-194-7889 \mathrm{~m}$ ). The voids are mainly distributed on the southwestern and southeastern borders, and are shown as the white pixels. The blue tracks indicate the spatial coverage of the ICESat data points in this region.

Similar to Section 4.2.1, we give the accuracy statistics for the global DEM products in the Xinjiang Uygur Autonomous Region in Table 4. For the void areas in the Xinjiang 
Uygur Autonomous Region, the accuracy of the SRTM-3 data is significantly affected by the anomalies, which results in the lower overall accuracy. The processed dataset outperforms both the ASTER GDEM and SRTM-1 data in terms of all the indexes used to measure the vertical accuracy. As the improvement is related to the spatial distribution of the voids and the ICESat reference data points, the related information is given in Fig. 15 . Furthermore, the specific accuracy assessment statistics for each void tile with sufficient data samples are displayed in Fig. 16. The improvement of the processed dataset can be clearly observed both in terms of both the evaluation results in the voids areas and the overall accuracy.

Table 4 The accuracy statistics in the Xinjiang Uygur Autonomous Region for the void pixels and the overall region, respectively.

\begin{tabular}{cccc|cccc}
\hline \hline \multirow{2}{*}{ Unit: $\mathrm{m}$} & \multicolumn{3}{c|}{ Void } & \multicolumn{4}{c}{ Overall } \\
\cline { 2 - 8 } & ASTER & SRTM-3 & Processed & ASTER & SRTM-3 & SRTM-1 & Processed \\
\hline MAE & 27.522 & 176.901 & 23.243 & 9.889 & 8.503 & 4.531 & 4.507 \\
\hline SD & 46.391 & 247.115 & 37.825 & 15.560 & 26.655 & 9.405 & 9.116 \\
\hline RMSE & 49.406 & 285.706 & 38.302 & 16.010 & 26.695 & 9.711 & 9.416 \\
\hline \hline
\end{tabular}

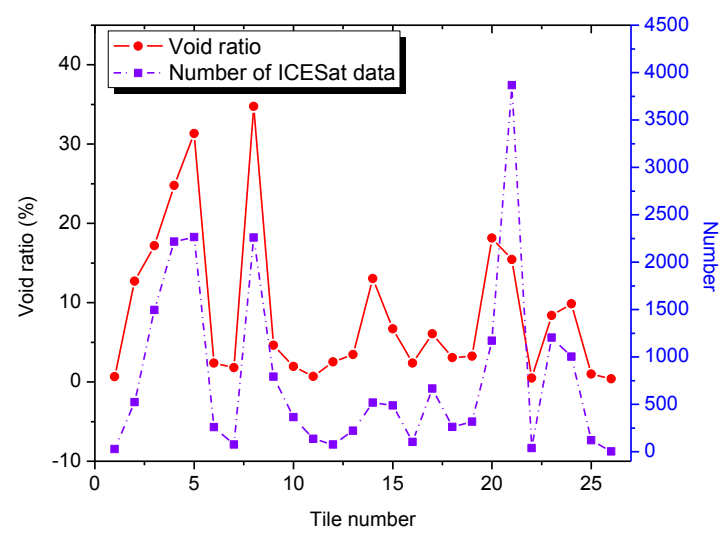

Fig. 15. Distribution of voids and ICESat points distributed within the voids in each void tile in the Xinjiang Uygur Autonomous Region. 

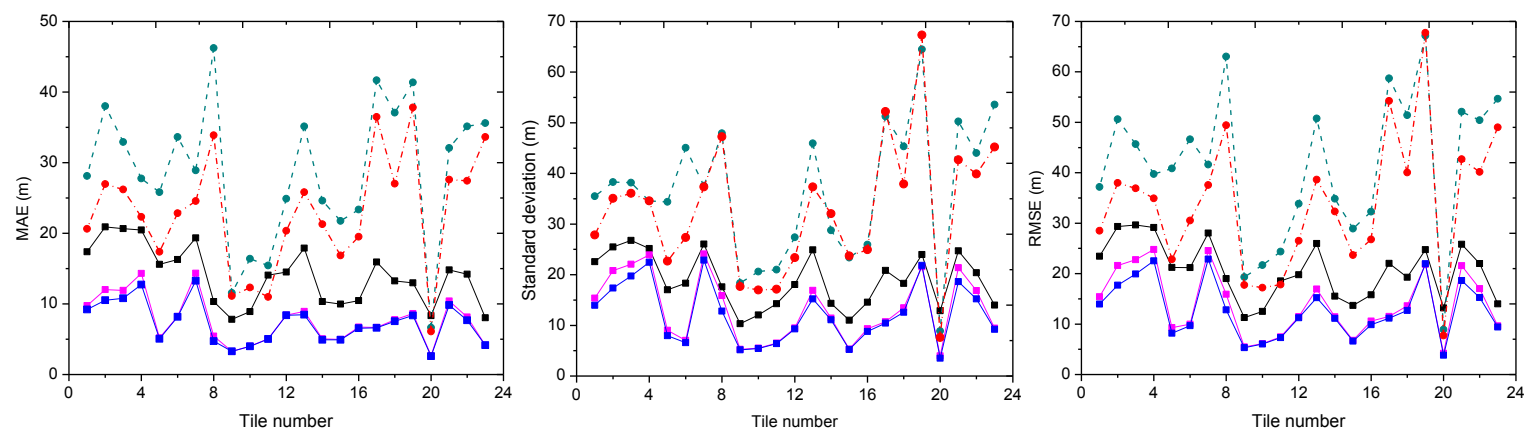

Fig. 16. The vertical accuracy assessment of the void data in the Xinjiang Uygur Autonomous Region. (a)-(c) indicate the MAE, SD, and RMSE values for the 23 tiles with voids, respectively.

The visual comparison is given in Figs. 17-18. The sample tiles in this region mainly consisted of desert, and $15.43 \%$ of the pixels were invalid. The ASTER GDEM data were clearly affected by noise and anomalies, as shown in Fig. 17(b). Over 25000 ICESat points were incorporated in the accuracy improvement and generated a seamless result with clearer terrain relief. The shaded relief map of the cropped region is given in Fig. 18 . Compared with the directly filled data, the merged result shows smoother transitions over the borders of the voids. Meanwhile, most of the anomalies in the original ASTER GDEM data are removed after the correction and filtering process.

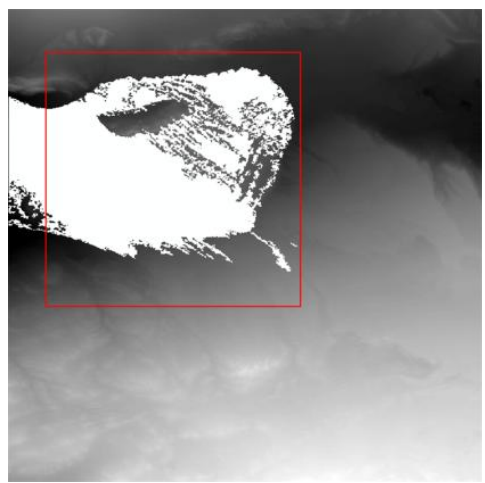

(a)

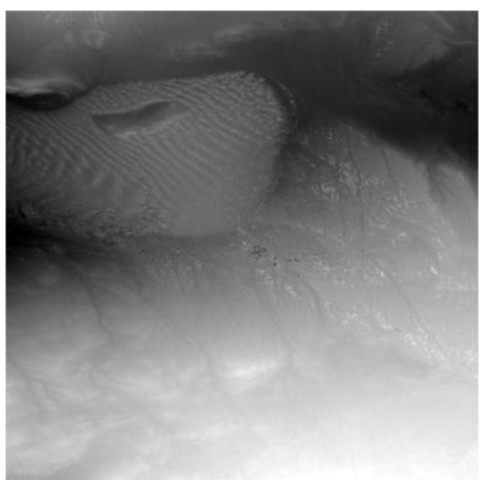

(b)

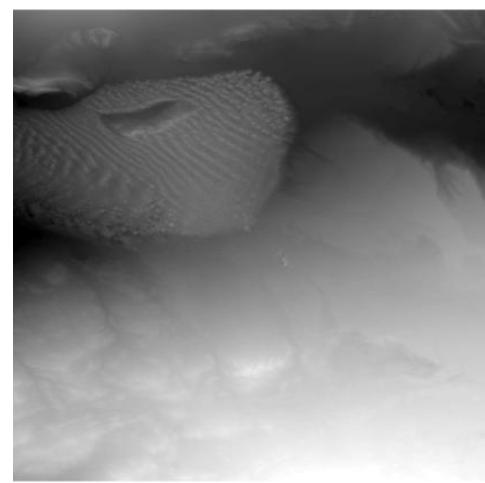

(c)

Fig. 17. A sample area in the Xinjiang Uygur Autonomous Region for visual inspection. (a)-(c) represent the original SRTM-1 data, the ASTER GDEM data, and the processed result. 


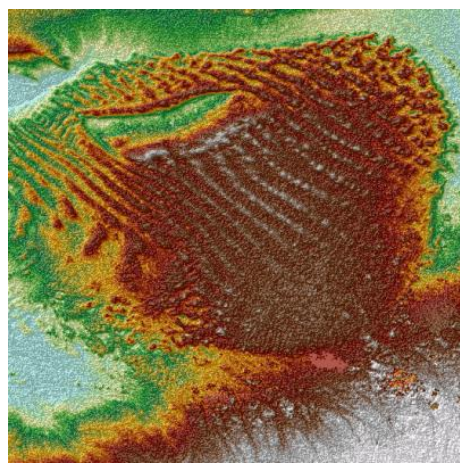

(a)

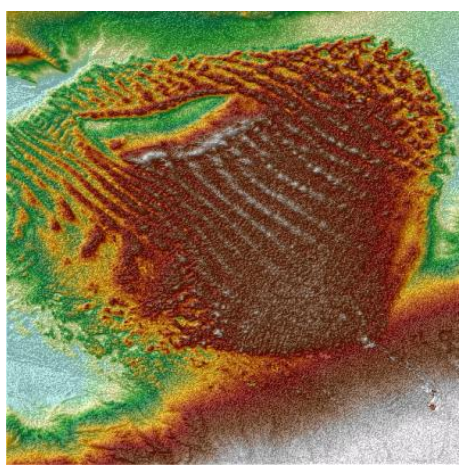

(b)

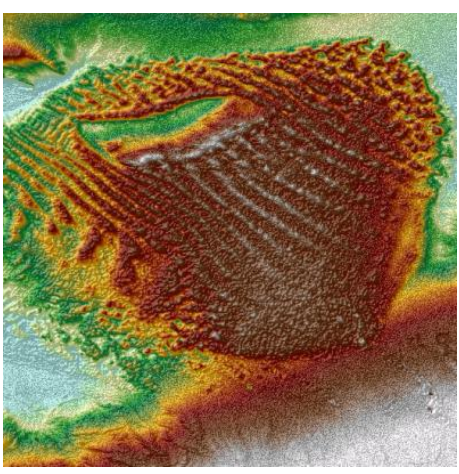

(c)

Fig. 18. The shaded relief map of the rectangular region in Fig. 16(a). (a)-(c) represent the ASTER GDEM data, the SRTM data filled directly using the ASTER GDEM values, and the processed result, respectively.

\subsection{Extracted channel network assessment}

One of the most significant applications of DEM data is hydrological information extraction. Therefore, we compared the river networks extracted from the global DEM products in the Yangtze River Basin, China. The Yangtze River is the longest river in Asia and the third-longest in the world, spanning over $6300 \mathrm{~km}$. It starts from the glaciers on the Tibet Plateau in Qinghai, and flows eastward across southwest, central, and eastern China. The sample area in Fig. 19 covers most of the drainage area, where the partially extracted stream flows are presented. We used the Arc Hydro Tools embedded in ArcGIS software to extract the drainage system in this study. The reference flow network (red) was provided by the National Science and Technology Infrastructure Program of China ${ }^{1}$. It corresponds to a resolution of $\sim 1 \mathrm{~km}$, with a high quality, after postprocessing by the Nanjing Institute of Geography and Limnology.

It can be seen in Fig. 19 that the network extracted from the SRTM data is denser than the reference due to the difference in the data resolutions. However, the two channel

\footnotetext{
1 The data are from the National Science and Technology Infrastructure Program of China, the Data Sharing Infrastructure of Earth System Science-Lake-Watershed Science Data Center (http://lake.data.ac.cn).
} 
networks are basically consistent in terms of the flow directions and branch locations. For a more detailed comparison, we provide zoomed views of the rectangular regions in Fig. 19, and the extracted flows from the SRTM-3 and ASTER GDEM data are also shown for comparison. In Fig. 20, Region (a) is a sample area with high mountains in Sichuan province. Therefore, there were large areas of voids in this data tile, which affected the continuity of the extracted channels. After void filling with the corrected height values, the extracted stream flows are continuous and similar to the reference data. As the ASTER GDEM was used as the source data in the filling process, the flows extracted from the GDEM and processed dataset are similar in the void regions. However, there are visible differences between them in the elliptical area of Fig. 20(a). The spurious branches due to the noises and subtle distortions in the ASTER GDEM terrain representation are successfully eliminated in the extracted channels from the processed data, which indicates the effectiveness of the proposed method. The hydrological information extracted from the high-resolution DEM products is much better than the that extracted from the low-resolution SRTM-3 data, especially over the relatively flat topography (Fig. 20(b)). Furthermore, it can be observed that the SRTM-1 data perform the best in hydrological information extraction in Fig. 20(c). 


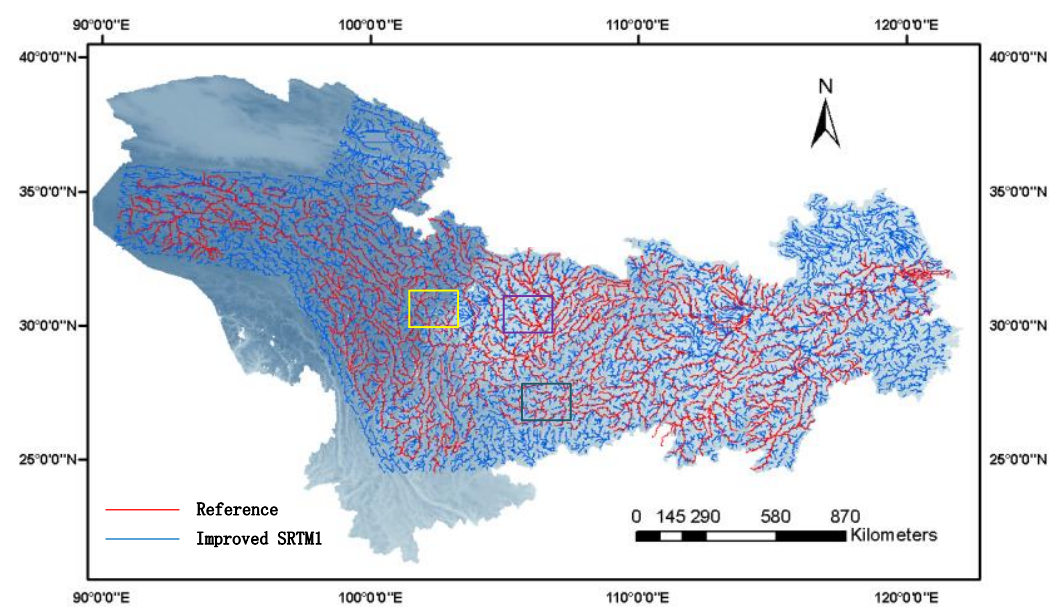

Fig. 19. The extracted river networks in the Yangtze River Basin, China. The extracted result by the processed dataset is shown in blue, with the red lines indicating the reference stream flow.

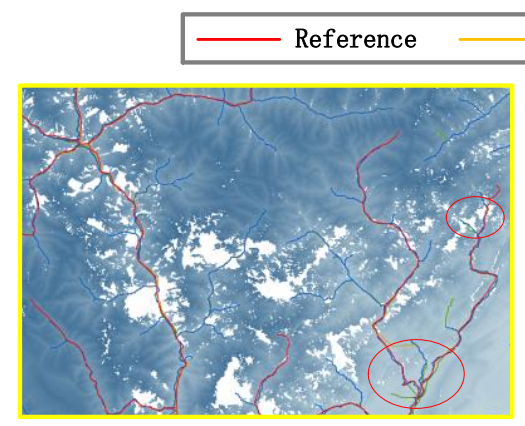

(a)

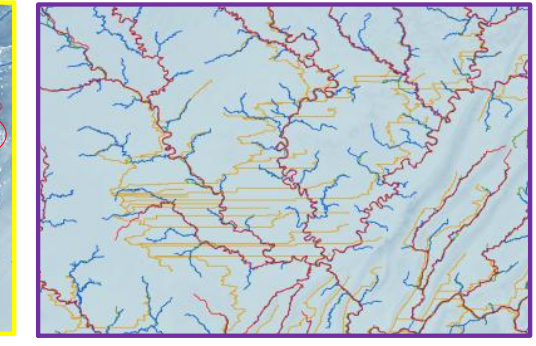

(b)
Processed dataset

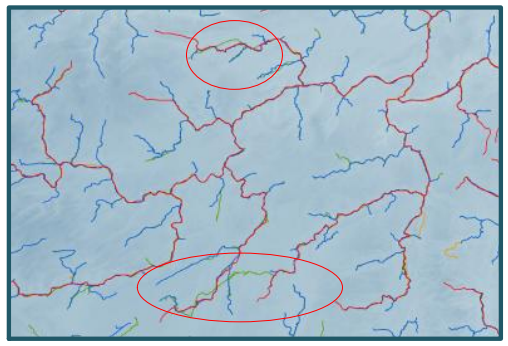

(c)

Fig. 20. Details of the three rectangular regions in Fig. 18. (a)-(c) are the zoomed displays of the above rectangular regions (with the same colors), respectively. The stream flows extracted by the SRTM-3 data (orange) and the ASTER GDEM (green) are also shown. The elliptical areas in (a) and (c) highlight the significant differences between the extracted flows in the sample areas.

\section{Conclusions and future work}

In this paper, we have addressed a method for the improvement of the global SRTM-1 DEM through the blending of the ASTER GDEM and ICESat laser altimetry data. The main problem with the recently released SRTM-1 data is the remaining voids. The processing flow of the proposed method can deal with the voids by incorporating the auxiliary data. Instead of filling the voids with other datasets directly, we utilize an ANN model to enhance the accuracy of the ASTER GDEM, using the ICESat data as reference points, considering the noise and anomalies in the ASTER data. Furthermore, the vertical 
bias between the elevation products in the multi-source data fusion is handled with a TIN-based delta surface method. Finally, an outlier filter is applied to all the data tiles.

An accuracy assessment was performed to validate the vertical accuracy of the corrected dataset, as well as the ASTER GDEM data, the SRTM-3 data, and the original SRTM-1 data. The currently processed dataset covers most of the eastern Asian continent; therefore, we mainly present the results from the Chinese mainland in this paper. It can be seen from the results that fused dataset shows a promising improvement in data quality compared with the SRTM-3 data and the ASTER GDEM. The voids existed in the original data are seamlessly filled, and the accuracy is improved compared with the other datasets in terms of the statistical assessment and visual performance. The results presented in this paper can also reflect the accuracy level of the global DEM datasets in other places with similar spatial patterns. After the data improvement, the new SRTM-1 data could be better used in applications such as hydrology and geology.

However, it is worth mentioning that the performance of the proposed framework depends on the distribution of the voids and the ICESat points. If we perform accuracy enhancement on the data tiles without sufficient training samples, the unreliable results might be obtained. The voids in China are mainly distributed in the western, where the density of the GLAS samples can satisfy our demands. In our work, this issue will be analyzed in detail when the remaining data tiles around the world are processed. Furthermore, the correction performance inevitably depends on the data quality to some extent. For example, the correction results might be influenced by the inaccurate slope information calculated using the ASTER data contaminated by the clouds and shadow. In 
the regions with large-scale cloud-covered and shaded observations, the artefacts are difficult to eliminate. However, as the ASTER GDEM data were generated with data scenes collected from 2000-2011, the repeating data reduce the influence of clouds. On the other hand, compared with the SRTM data generated with SAR observations, the accuracy of the ASTER GDEM derived from optical data and ICESat laser scanning points are less sensitive to the slope angles.

The final processed result comprises seamless SRTM-1 land elevation data covering $60^{\circ} N$ to $\sim 56^{\circ} S$ globally. The finished data have been published on an FTP server at http://sendimage.whu.edu.cn/res/DEM_share/. The remaining data will be processed in each $30^{\circ} \times 30^{\circ}$ grid area over a global extent. The latest status of the processing will also be published on the website. The global data are expected to be finished by the end of 2016 . Due to the limited validation data source, we only undertook an accuracy assessment based on the ICESat data. Further validation with other reliable reference data would help to allow a more comprehensive understanding of the processed dataset.

\section{Acknowledgement}

This research was supported by the National Natural Science Foundation of China (41422108), the Cross-disciplinary Collaborative Teams Program for Science, Technology and Innovation of the Chinese Academy of Sciences and the Hongkong Scholars Program under Grant XJ2014009. We gratefully thank the data distribution agencies who provided the publicly released data used in this work. The SRTM-1 and ASTER GDEM v2 data were obtained from the USGS EarthExplorer site (http://earthexplorer.usgs.gov/ ), and the 
SRTM3 v4.1 data can be obtained online through the CGIAR-CSI download database at http://srtm.csi.cgiar.org/. The ICESat GLAS 14 data were provided by the National Snow and Ice Data Center (NSIDC) (http://nsidc.org/data/icesat/order.html). The service from NSIDC was helpful for us in processing the ICESat points. Furthermore, we are grateful for the support of the Lake-Watershed Science Data Center, National Earth System Science Data Sharing Infrastructure, National Science and Technology Infrastructure of China (http://lake.geodata.cn).

Lastly, the authors would like to thank the editors and the anonymous reviewers for their valuable suggestions. Their insightful comments helped us make a major improvement to this manuscript.

\section{References}

(2014). WorldDEM: The New Standard of Global Elevation Models. Retrieved from http://www.intelligence-airbusds.com/files/pmedia/public/r5434_9_int_022_worlddem_en_low.pdf.

Abshire, J. B., X. Sun, H. Riris, J. M. Sirota, J. F. McGarry, S. Palm, D. Yi and P. Liiva, 2005. Geoscience laser altimeter system (GLAS) on the ICESat mission: on- orbit measurement performance. Geophysical Research Letters 32(21).

Arefi, H. and P. Reinartz, 2011. Accuracy enhancement of ASTER global digital elevation models using ICESat data. Remote Sensing 3(7): 1323-1343.

Athmania, D. and H. Achour, 2014. External validation of the ASTER GDEM2, GMTED2010 and CGIAR-CSI-SRTM v4. 1 free access digital elevation models (DEMs) in Tunisia and Algeria. Remote Sensing 6(5): 4600-4620.

Baghdadi, N., N. Lemarquand, H. Abdallah and J. S. Bailly, 2011. The relevance of GLAS/ICESat elevation data for the monitoring of river networks. Remote Sensing 3(4): 708-720.

Baselice, F., G. Ferraioli and V. Pascazio, 2009. DEM reconstruction in layover areas from SAR and auxiliary input data. Geoscience and Remote Sensing Letters, IEEE 6(2): 253-257.

Bhang, K. J., F. W. Schwartz and A. Braun, 2007. Verification of the vertical error in C-band SRTM DEM using ICESat and Landsat-7, Otter Tail County, MN. Geoscience and Remote Sensing, IEEE Transactions on 45(1): 36-44.

Crosetto, M., 2002. Calibration and validation of SAR interferometry for DEM generation. ISPRS Journal of 
Photogrammetry and Remote Sensing 57(3): 213-227.

Dowding, S., T. Kuuskivi and X. Li, 2004. Void fill of SRTM elevation data-principles, processes and performance. ASPRS Images to Decision: Remote Sensing Foundation for GIS Applications: 12-16.

Farr, T. G. and M. Kobrick, 2000. Shuttle Radar Topography Mission produces a wealth of data. Eos, Transactions American Geophysical Union 81(48): 583-585.

Fritz, T., H. Breit, C. Rossi, U. Balss, M. Lachaise and S. Duque, 2012. Interferometric processing and products of the TanDEM-X mission. Geoscience and Remote Sensing Symposium (IGARSS), 2012 IEEE International, 1904-1907, IEEE.

Fu, P. and P. M. Rich, 2002. A geometric solar radiation model with applications in agriculture and forestry. Computers and electronics in agriculture 37(1): 25-35

Gamache, M., 2004. Free and low cost datasets for international mountain cartography. 4th ICA Mountain Cartography Workshop, 26.

González, J. H., M. Bachmann, R. Scheiber and G. Krieger, 2010. Definition of ICESat selection criteria for their use as height references for TanDEM-X. Geoscience and Remote Sensing, IEEE Transactions on 48(6): 2750-2757.

Grohman, G., G. Kroenung and J. Strebeck, 2006. Filling SRTM voids: The delta surface fill method. Photogrammetric Engineering and Remote Sensing 72(3): 213-216.

Hengl, T. and H. Reuter, 2011. How accurate and usable is GDEM? A statistical assessment of GDEM using LiDAR data. Geomorphometry 2: 45-48.

Hirt, C., M. Filmer and W. Featherstone, 2010. Comparison and validation of the recent freely available ASTER-GDEM ver1, SRTM ver4. 1 and GEODATA DEM-9S ver3 digital elevation models over Australia. Australian Journal of Earth Sciences 57(3): 337-347.

Hsu, K. 1., H. V. Gupta and S. Sorooshian, 1995. Artificial neural network modeling of the rainfall- runoff process. Water Resources Research 31(10): 2517-2530.

Huber, M., B. Wessel, D. Kosmann, A. Felbier, V. Schwieger, M. Habermeyer, A. Wendleder and A. Roth, 2009. Ensuring globally the TanDEM-X height accuracy: Analysis of the reference data sets ICESat, SRTM and KGPS-Tracks. Geoscience and Remote Sensing Symposium, 2009 IEEE International, IGARSS 2009, II-769-II-772, IEEE.

Jacobsen, K., 2010. Comparison of ASTER GDEMs with SRTM height models. EARSeL Symposium, Dubrovnik. Remote Sensing for Science, Education and Natural and Cultural Heritage, 521-526.

Jarvis, A., H. I. Reuter, A. Nelson and E. Guevara, 2008. Hole-filled SRTM for the globe Version 4. available from the CGIAR-CSI SRTM 90m Database (http://srtm. csi. cgiar. org).

Jia, B., Z. Zhang, L. Ci, Y. Ren, B. Pan and Z. Zhang, 2004. Oasis land-use dynamics and its influence on the oasis environment in Xinjiang, China. Journal of Arid Environments 56(1): 11-26.

Karkee, M., B. L. Steward and S. A. Aziz, 2008. Improving quality of public domain digital elevation models through data fusion. Biosystems Engineering 101(3): 293-305.

Kellndorfer, J., W. Walker, L. Pierce, C. Dobson, J. A. Fites, C. Hunsaker, J. Vona and M. Clutter, 2004. Vegetation height estimation from shuttle radar topography mission and national elevation datasets. Remote Sensing of Environment 93(3): 339-358.

Li, P., C. Shi, Z. Li, J.-P. Muller, J. Drummond, X. Li, T. Li, Y. Li and J. Liu, 2013. Evaluation of ASTER GDEM using GPS benchmarks and SRTM in China. International Journal of Remote Sensing 34(5): 1744-1771.

Ling, F., Q. Zhang and C. Wang, 2007. Filling voids of SRTM with Landsat sensor imagery in rugged terrain. International Journal of Remote Sensing 28(2): 465-471. 
Luedeling, E., S. Siebert and A. Buerkert, 2007. Filling the voids in the SRTM elevation model-A TIN-based delta surface approach. ISPRS Journal of Photogrammetry and Remote Sensing 62(4): 283-294.

Luk, K., J. Ball and A. Sharma, 2000. A study of optimal model lag and spatial inputs to artificial neural network for rainfall forecasting. Journal of Hydrology 227(1): 56-65.

Ma, Y., S. Kang, L. Zhu, B. Xu, L. Tian and T. Yao, 2008. Roof of the world: Tibetan observation and research platform: Atmosphere-land Interaction over a heterogeneous landscape. Bulletin of the American Meteorological Society 89(10): 1487-1492.

Mukherjee, S., P. Joshi, S. Mukherjee, A. Ghosh, R. Garg and A. Mukhopadhyay, 2013. Evaluation of vertical accuracy of open source Digital Elevation Model (DEM). International Journal of Applied Earth Observation and Geoinformation 21: 205-217.

Næsset, E., H. O. Ørka, S. Solberg, O. M. Bollandsås, E. H. Hansen, E. Mauya, E. Zahabu, R. Malimbwi, N. Chamuya and H. Olsson, 2016. Mapping and estimating forest area and aboveground biomass in miombo woodlands in Tanzania using data from airborne laser scanning, TanDEM-X, RapidEye, and global forest maps: A comparison of estimated precision. Remote Sensing of Environment 175 : 282-300.

NSIDC. (2014). GLAH14 Product Data Dictionary. Retrieved 13 March, 2014, from http://nsidc.org/data/docs/daac/glas_altimetry/data-dictionary-glah14.html.

Öztopal, A., 2006. Artificial neural network approach to spatial estimation of wind velocity data. Energy Conversion and Management 47(4): 395-406.

Pipaud, I., D. Loibl and F. Lehmkuhl, 2015. Evaluation of TanDEM-X elevation data for geomorphological mapping and interpretation in high mountain environments-A case study from SE Tibet, China. Geomorphology 246: 232-254.

Reuter, H. I., A. Nelson and A. Jarvis, 2007. An evaluation of void- filling interpolation methods for SRTM data. International Journal of Geographical Information Science 21(9): 983-1008.

Ripley, B. D., 2007. Pattern recognition and neural networks. City, Cambridge university press.

Robinson, N., J. Regetz and R. P. Guralnick, 2014. EarthEnv-DEM90: A nearly-global, void-free, multi-scale smoothed, 90m digital elevation model from fused ASTER and SRTM data. ISPRS Journal of Photogrammetry and Remote Sensing 87(0): 57-67.

Satgé, F., M.-P. Bonnet, F. Timouk, S. Calmant, R. Pillco, J. Molina, W. Lavado-Casimiro, A. Arsen, J. Crétaux and J. Garnier, 2015. Accuracy assessment of SRTM v4 and ASTER GDEM v2 over the Altiplano watershed using ICESat/GLAS data. International Journal of Remote Sensing 36(2): 465-488.

Schutz, B., H. Zwally, C. Shuman, D. Hancock and J. DiMarzio, 2005. Overview of the ICESat mission. Geophysical Research Letters 32(21).

Tachikawa, T., M. Hato, M. Kaku and A. Iwasaki, 2011. Characteristics of ASTER GDEM version 2. Geoscience and Remote Sensing Symposium (IGARSS), 2011 IEEE International, 3657-3660, IEEE.

Tachikawa, T., M. Kaku, A. Iwasaki, D. Gesch, M. Oimoen, Z. Zhang, J. Danielson, T. Krieger, B. Curtis and J. Haase, 2011. ASTER Global Digital Elevation Model Version 2-Summary of Validation Results. ASTER GDEM Validation Team (http://www._jspacesystems. or. jp/ersdac/GDEM/ver2Validation/Summary_GDEM2_v alidation_report_final. pdf).

Tadono, T., J. Takaku, K. Tsutsui, F. Oda and H. Nagai, 2015. Status of “ALOS World 3D (AW3D)" global DSM generation. Geoscience and Remote Sensing Symposium (IGARSS), 2015 IEEE International, 
3822-3825, IEEE.

Team, A. V., 2009. ASTER global DEM validation summary report. METI \& NASA. 28pp.

Tighe, M. L. and D. Chamberlain, 2009. Accuracy comparison of the SRTM, ASTER, NED, NEXTMap® USA Digital Terrain Model over several USA study sites. Proceedings of the ASPRS/MAPPS fall conference.

Toutin, T., 2002. Impact of terrain slope and aspect on radargrammetric DEM accuracy. ISPRS Journal of Photogrammetry and Remote Sensing 57(3): 228-240.

USGS. (1996). Retrieved, from https://lta.cr.usgs.gov/GTOPO30.

USGS. (2010). Retrieved, from https://lta.cr.usgs.gov/GMTED2010.

Van Zyl, J. J., 2001. The Shuttle Radar Topography Mission (SRTM): a breakthrough in remote sensing of topography. Acta Astronautica 48(5): 559-565.

Verdin, A., B. Rajagopalan, W. Kleiber and C. Funk, 2015. A Bayesian kriging approach for blending satellite and ground precipitation observations. Water Resources Research 51(2): 908-921.

Wang, L. and K. Wang, 2015. Impacts of DEM uncertainty on estimated surface solar radiation and extracted river network. Bulletin of the American Meteorological Society 96(2): 297-304.

Wechsler, S., 2007. Uncertainties associated with digital elevation models for hydrologic applications: a review. Hydrology and Earth System Sciences Discussions 11(4): 1481-1500.

Yang, L., X. Meng and X. Zhang, 2011. SRTM DEM and its application advances. International Journal of Remote Sensing 32(14): 3875-3896.

Yang, X., Z. Zhu, D. Jaekel, L. Owen and J. Han, 2002. Late Quaternary palaeoenvironment change and landscape evolution along the Keriya River, Xinjiang, China: the relationship between high mountain glaciation and landscape evolution in foreland desert regions. Quaternary International 97: 155-166.

Yue, L., W. Yu, H. Shen, L. Zhang and Y. He, 2015. Accuracy assessment of SRTM V4. 1 and ASTER GDEM V2 in high-altitude mountainous areas: A case study in Yulong Snow Mountain, China. Geoscience and Remote Sensing Symposium (IGARSS), 2015 IEEE International, 5011-5014, IEEE.

Yue, T.-X., C.-F. Chen and B.-L. Li, 2012. A high-accuracy method for filling voids and its verification. International Journal of Remote Sensing 33(9): 2815-2830.

Zhang, G., H. Xie, S. Kang, D. Yi and S. F. Ackley, 2011. Monitoring lake level changes on the Tibetan Plateau using ICESat altimetry data (2003-2009). Remote Sensing of Environment 115(7): 1733-1742.

Zhang, L., K. Wu, Y. Zhong and P. Li, 2008. A new sub-pixel mapping algorithm based on a BP neural network with an observation model. Neurocomputing 71(10): 2046-2054.

Zhao, S., W. Cheng, C. Zhou, X. Chen, S. Zhang, Z. Zhou, H. Liu and H. Chai, 2011. Accuracy assessment of the ASTER GDEM and SRTM3 DEM: an example in the Loess Plateau and North China Plain of China. International Journal of Remote Sensing 32(23): 8081-8093.

Zheng, X., H. Xiong, J. Gong and L. Yue, 2015. A robust channel network extraction method combining discrete curve evolution and the skeleton construction technique. Advances in Water Resources 83: $17-27$. 